\title{
THE HASSE PRINCIPLE FOR LINES ON DEL PEZZO SURFACES
}

\author{
JÖRG JAHNEL AND DANIEL LOUGHRAN
}

\begin{abstract}
In this paper, we consider the following problem: Does there exist a cubic surface over $\mathbb{Q}$ which contains no line over $\mathbb{Q}$, yet contains a line over every completion of $\mathbb{Q}$ ? This question may be interpreted as asking whether the Hilbert scheme of lines on a cubic surface can fail the Hasse principle. We also consider analogous problems, over arbitrary number fields, for other del Pezzo surfaces and complete intersections of two quadrics.
\end{abstract}

\section{Contents}

1. Introduction

2. 0-dimensional schemes and del Pezzo surfaces

3. The Hasse principle for lines on del Pezzo surfaces

4. Distribution of counter-examples

5. Intersections of two quadrics

References

\section{INTRODUCTION}

One says that a class of varieties over a number field $k$ satisfies the Hasse principle if for each variety in the class, the existence of a rational point over every completion of $k$ implies the existence of a rational point over $k$. This principle takes its name from the classical Hasse-Minkowski theorem, which states that the Hasse principle holds for the class of quadric hypersurfaces. Already for cubic curves and cubic surfaces however, the Hasse principle can fail. There has been much work on constructing and controlling such failures, and moreover there are many positive results proving that the Hasse principle holds for special classes of varieties. For an overview of various results and methods, see [44].

Recently, there has been an interest in Hasse-type principles for other existence problems in arithmetic geometry (e.g. isogenies of elliptic curves [48], [1] and Apollonian gaskets [6]). In this paper we study Hasse-type principles for linear subspaces on varieties. Namely, given a class of varieties embedded into a fixed projective space $\mathbb{P}^{n}$ over $k$ and some $r \in \mathbb{N}$, whether the existence of a linear subspace of dimension $r$ over every completion of $k$ guarantees the existence of a linear subspace of dimension $r$ over $k$. Note that this is in fact a special case of the usual Hasse principle. Indeed, there is a Hilbert scheme parametrising the

2010 Mathematics Subject Classification. 11G35 (primary), 11R32, 14J26, 14-04 (secondary). 
linear subspaces of fixed dimension, and we are asking whether these schemes satisfy the Hasse principle.

For complete intersections of hypersurfaces of odd degree in $\mathbb{P}^{n}$ over $k$, Birch [3] used the circle method to show that there always exists a linear subspace over $k$ of given dimension $r$, whenever $n$ is sufficiently large in terms of $r$ and the degrees of the equations (see [12] and [7] for recent improvements). There does not appear however to have been any work into such problems for varieties of small dimension. For example, as already noted, the Hasse principle can fail for cubic surfaces, but can the Hasse principle for lines fail for cubic surfaces?

Any smooth cubic surface over an algebraically closed field contains 27 lines. Moreover the Hilbert scheme of lines is reduced, hence it is a 0-dimensional scheme of degree 27. It is well-known that 0-dimensional schemes can fail the Hasse principle; counter-examples occur as the zero sets of monic integral polynomials with a root modulo every integer, but no integer root (such polynomials automatically have a real root; see Lemma 2.2). An explicit example here being

$$
\left(x^{2}-2\right)\left(x^{2}-17\right)\left(x^{2}-34\right)=0 .
$$

See [2], 46] and [47] for other examples. Our first result states that such schemes can indeed occur as the Hilbert scheme of lines on a cubic surface.

Theorem 1.1. Let $k$ be a number field. Then there exist smooth cubic surfaces over $k$ which fail the Hasse principle for lines.

Our proof is constructive. For example, an explicit counter-example $S \subset \mathbb{P}^{3}$ over $\mathbb{Q}$ is given by

$$
\begin{aligned}
& -5 x^{2} w-5 x y^{2}-2 x y w+5 x z^{2}-9 x z w-5 x w^{2}+9 y^{3}-11 y^{2} z+29 y^{2} w \\
& +43 y z^{2}-52 y z w-4 y w^{2}-13 z^{3}+14 z^{2} w-96 z w^{2}+45 w^{3}=0 .
\end{aligned}
$$

The reader may easily verify this assertion herself with some help from a computer. There is a univariate polynomial $f \in \mathbb{Q}[t]$ of degree 27 , the roots of which rationally parametrise the set of the 27 lines on the surface over $\overline{\mathbb{Q}}$ (namely $f(t)=0$ is isomorphic to the Hilbert scheme of lines on $S$ ). The polynomial $f$ may be determined explicitly, using Gröbner bases. It turns out that $f$ factorises into 4 irreducible polynomials $f=f_{1} f_{2} f_{3} f_{4}$ of degrees $2,5,10$, and 10, respectively. In particular, there is no $\mathbb{Q}$-rational line on $S$.

The fields defined by $f_{1}$ and $f_{2}$ are isomorphic to $\mathbb{Q}(\sqrt{5})$ and $\mathbb{Q}(\sqrt[5]{101})$, respectively. Hence $f_{1}$ has a root over $\mathbb{Q}_{p}$ if $p \equiv 1,4 \bmod 5$ or $p=\infty$, and $f_{2}$ has a root over $\mathbb{Q}_{p}$ if $p \equiv 2,3,4 \bmod 5$ or $p=5$. Thus $S$ contains a line over every completion of $\mathbb{Q}$, i.e. it is a counter-example to the Hasse principle for lines. We will explain in the main body of this article how we found this surface, and give a general method for constructing similar counter-examples. To the authors' knowledge, this is the first known counter-example to the Hasse principle for the Hilbert scheme of linear subspaces of positive dimension on a hypersurface.

Note that a cubic surface contains a line over $k$ if and only if it contains a conic over $k$. We therefore obtain the following amusing corollary to Theorem 1.1.

Corollary 1.2. Let $k$ be a number field. Then there exist smooth cubic surfaces over $k$ which fail the Hasse principle for conics. 
Again to the authors' knowledge, this is the first result of its kind in the literature. We are also able to prove results about the distribution of counterexamples. To state this, note that the coefficients of a cubic surface determine a point in $\mathbb{P}^{19}$. We therefore define the height of a cubic surface over $k$ to be the usual naive height of the corresponding point in $\mathbb{P}^{19}$ (see e.g. [38, §2]).

Theorem 1.3. Let $k$ be a number field.

(a) The collection of smooth cubic surfaces over $k$ which fail the Hasse principle for lines is Zariski dense in $\mathbb{P}^{19}$.

(b) Nevertheless, when ordered by the height of their coefficients, $100 \%$ of smooth cubic surfaces satisfy the Hasse principle for lines.

We prove Zariski density by showing that the set of the reductions of the counter-examples modulo suitable prime ideals of $k$ is rather large. The second part of this theorem follows from the fact that $100 \%$ of cubic surfaces have an irreducible Hilbert scheme of lines, together with the fact that irreducible finite étale schemes satisfy the Hasse principle (see Lemma 2.3).

Cubic surfaces belong to a natural class of surfaces, namely they are the del Pezzo surfaces of degree 3. Our next result solves the analogous problem for del Pezzo surfaces in general, where one has a natural notion of a line (see \$2.2.2).

Theorem 1.4. Let $k$ be a number field and let $1 \leq d \leq 9$. Then the class of del Pezzo surfaces of degree $d$ fails the Hasse principle for lines if and only if

$$
d=8,5,3,2 \text { or } 1 \text {. }
$$

Our results are actually stronger than stated here. Namely, we classify all possible cases where counter-examples can occur in terms of Galois actions on lines, and show that, at least when $d \geq 3$, counter-examples of these types actually exist. We also prove an analogue of Theorem 1.3, by showing that the collection of counter-examples is a Zariski dense, yet thin, subset of a suitable Hilbert scheme (see Theorem 4.2). This is one of the main results in this paper, and it says that whilst there are many counter-examples, they are still in some sense quite rare. See 33 and $\$ 4$ for our complete results on del Pezzo surfaces.

We now explain the ideas behind the proof of Theorem 1.4. The cases of degrees 9 and 8 are handled using some classical geometric methods. For the other cases, we use the Chebotarev density theorem to give an explicit criterion on the Galois group of the splitting field of the surface, in terms of its action on the graph of lines of the surface, for the surface to be a counter-example to the Hasse principle for lines. This reduces the problem to a purely group theoretic one about classifying certain group actions on certain graphs, which we perform using a computer. In the case of degree 7,6 or 4 , one finds that this criterion is never satisfied, hence the Hasse principle for lines always holds.

In degrees 5,3,2 and 1, we find explicit groups which satisfy our criterion. A priori however, there is no guarantee that there exist del Pezzo surfaces with these Galois actions over $k$ (this is the "inverse Galois problem" for del Pezzo surfaces). In degrees 5 and 3, there are very few cases, and moreover the surfaces which arise this way are rational. Thus we construct surfaces with these Galois actions by performing blow-ups and blow-downs of configurations of closed points in $\mathbb{P}^{2}$. These geometrical constructions yield surfaces with a line over all but finitely 
many completions of $k$, yet no line over $k$. To obtain counter-examples to the Hasse principle for lines, we need quite strict control over the decomposition groups of the splitting fields of the blown-up points. When the Galois group is solvable, this is achieved using results of Sonn [46]. The only non-solvable group which occurs is $S_{5}$, and here we use work of Kedlaya on the construction of quintic fields with square-free discriminants [23]. For del Pezzo surfaces of degrees 2 and 1 many more cases arise. We therefore illustrate the range of behaviour which occurs by constructing both rational and minimal counter-examples (i.e. surfaces for which no Galois orbit of lines can be contracted). These latter surfaces arise as conic bundles.

Note that Theorem 1.4 implies that del Pezzo surfaces of degree 4 satisfy the Hasse principle for lines, something which is by no means obvious. The authors were able to find a non-computer-assisted proof of this result, and moreover generalise it to intersections of two quadrics of even dimension.

Theorem 1.5. Let $n \geq 0$ and let $k$ be a number field. Let $X$ be a smooth $2 n$-dimensional complete intersection of two quadrics over $k$. If $X$ contains no $n$-plane over $k$, then it contains no n-plane over infinitely many completions of $k$. In particular, $X$ satisfies the Hasse principle for $n$-planes.

The planes considered in Theorem 1.5 are in some respects the most interesting, since they are the linear subspaces of maximal dimension which can occur. There are various results already in the literature on the existence of linear subspaces on intersections of quadrics. For example, an application of the version of the circle method used in [7] would prove the Hasse principle for $r$-planes when $r$ has order of magnitude $O(\sqrt{n})$ and $k=\mathbb{Q}$. Much more is known when $k$ is totally imaginary; namely, as explained in [13, (2.11)], one may combine [9, Cor. 10.4], [29, Cor. 2.4] and [32, Thm. 1] to obtain the existence of an $r$-plane here for $r$ of the size $2 n / 3+O(1)$. In particular, Theorem [1.5 is out of reach from what these methods are currently able to achieve. It is proved by showing that the Hilbert scheme of $n$-planes on $X$ is a torsor for a group scheme whose Tate-Shafarevich group is trivial.

The analogue of Theorem 1.5 fails for smooth $(2 n+1)$-dimensional complete intersections of two quadrics over $k$ (the linear subspaces of maximal dimension being $n$-planes). Here the Hilbert scheme of $n$-planes is a torsor for the intermediate Jacobian of $X$. For $n=0$, we obtain a curve of genus 1 , and it is well-known that these can fail the Hasse principle (see [44, p. 164] for an explicit example over $\mathbb{Q}$ ). In private communication, Damiano Testa has informed the authors that he is able to construct counter-examples of dimension 3 over $\mathbb{Q}$.

Outline of the paper. In Section 2 we gather results on the Hasse principle for finite étale schemes and the structure of the Hilbert scheme of lines on del Pezzo surfaces. Theorem 1.4 is then proved in Section 3. Theorem 1.3, together with its generalisation Theorem 4.2, is proved in Section 4. Section 5 is dedicated to the proof of Theorem 1.5.

Acknowledgements. The authors have benefited from conversations with Julia Brandes, Jean-Louis Colliot-Thélène, Rainer Dietmann, Jordan Ellenberg, 
Andreas-Stephan Elsenhans, Christopher Frei, David Speyer, Anthony VárillyAlvarado and Trevor Wooley. All computations are with Magma [4].

1.1. Notation. For a scheme $X$ of finite type over a field $k$, we denote by $\pi_{0}(X)$ the scheme of connected components of $X$ (see [30, Def. 10.2.19]). For a field extension $k \subset K$, we denote by $X_{K}=X \times_{k} K$ the base change of $X$ to $K$.

For a number field $k$, we denote its ring of integers by $\mathcal{O}_{k}$. For a place $v$ of $k$, we shall denote by $k_{v}$ the completion of $k$ with respect to $v$. For a non-zero prime ideal $\mathfrak{p}$ of $k$, we denote by $\mathbb{F}_{\mathfrak{p}}$ the corresponding residue field and by $\mathcal{O}_{k, \mathfrak{p}}$ the localisation of $\mathcal{O}_{k}$ at $\mathfrak{p}$.

Bold math letters (e.g. $\mathbf{A}_{n}$ ) are used for root systems, while for groups we use non-bold math letters (e.g. $A_{n}$ is the alternating group on $n$ letters).

\section{0-Dimensional SChemes And Del Pezzo Surfaces}

We begin with some results on the arithmetic of finite étale schemes. Our interest in these stems from the fact that they occur as the Hilbert schemes of lines on del Pezzo surfaces. We finish this section by gathering some results on the geometry of del Pezzo surfaces, together with results on their Hilbert schemes and graphs of lines.

2.1. Finite étale schemes. Let $X$ be a finite étale scheme over a perfect field $k$ (i.e. $X$ is a finite reduced 0-dimensional scheme over $k$ ). We define the degree of $X$ to be

$$
\operatorname{deg} X=\sum_{x \in X}[k(x): k],
$$

where the sum is over all points $x$ of $X$, and $k(x)$ denotes the residue field of $x$. We shall say that such a scheme $X$ is split if

$$
\# X(k)=\operatorname{deg} X .
$$

The smallest field $K$ over which $X$ becomes split is called the splitting field of $X$. This is a finite Galois extension of $k$.

2.1.1. A dictionary. There is a useful well-known dictionary for such schemes. Namely, let $K / k$ be a finite Galois extension with Galois group $\Gamma$ and let $X$ be a finite étale scheme over $k$ with splitting field $K$. Firstly, to $X$ one may associate the $\Gamma$-set (i.e. a set with a left action of $\Gamma$ ) given by $X(K)$ equipped with its natural $\Gamma$-action. Secondly, we may also associate to $X$ the finite étale $k$-algebra

$$
\prod_{x \in X} k(x)
$$

These constructions give rise to bijections between the following sets:

(1) The set of $k$-isomorphism classes of finite étale schemes over $k$ with splitting field $K$.

(2) The set of $k$-isomorphism classes of finite étale $k$-algebras with splitting field $K$.

(3) The set of $\Gamma$-isomorphism classes of finite $\Gamma$-sets with a faithful action of $\Gamma$. 
See [5, Thm. V.6.4] and [5, Prop. V.10.12]. We shall frequently use this dictionary to pass between these different objects in this paper. One trivial, though key, property is that $X$ admits a $k$-point if and only if $\Gamma$ acts with a fixed point on the associated $\Gamma$-set $X(K)$.

2.1.2. The Hasse principle. We now gather some mostly well-known results on the Hasse principle for finite étale schemes, via the use of our dictionary. Throughout the remainder of this section $k$ is a number field. We begin with a criterion for local solubility at a given place.

Lemma 2.1. Let $X$ be a finite étale scheme over $k$. Let $K / k$ denote the splitting field, with Galois group $\Gamma$, and let $v$ be a place of $k$. Then $X$ admits a $k_{v}$-point if and only for any place $w$ of $K$ above $v$, the decomposition group of $w$ acts with a fixed point on the associated $\Gamma$-set $X(K)$.

Proof. The splitting field of $X_{k_{v}}$ is $K_{w}$, and the Galois group $\operatorname{Gal}\left(K_{w} / k_{v}\right)$ may be identified with the decomposition group of $w$. As $X$ admits a $k_{v}$-point if and only if this group acts with a fixed point, the result is proved.

Using this, we give a criterion for solubility at almost all places.

Lemma 2.2. Let $X$ be a finite étale scheme over $k$. Let $K / k$ denote the splitting field, with Galois group $\Gamma$. Then $X$ is locally soluble at all but finitely many places of $k$, but not soluble over $k$, if and only if on the associated $\Gamma$-set $X(K)$, each conjugacy class of $\Gamma$ acts with a fixed point, but the group $\Gamma$ acts without a fixed point.

In which case, for each place $v$ of $k$ which is either archimedean or unramified in $K$, the scheme $X$ admits a $k_{v}$-point.

Proof. The Chebotarev density theorem [34, Thm. 13.4] implies that every cyclic subgroup of $\Gamma$ occurs as a decomposition group for infinitely many places of $k$. Hence if there is an element of $\Gamma$ which acts without a fixed point, then by Lemma 2.1 there are infinitely many places $v$ of $k$ for which $X$ has no $k_{v}$-point. If however every conjugacy class of $\Gamma$ acts with a fixed point, then an application of Lemma 2.1 shows that $X$ admits a $k_{v}$-point for every place $v$ of $k$ which is either archimedean or unramified in $K / k$. This completes the proof.

We now present some simple cases in which the Hasse principle holds.

Lemma 2.3. Let $X$ be a finite étale scheme over $k$ and let $\Gamma$ denote the Galois group of the splitting field of $X$. Assume that $X$ is irreducible or that $\Gamma$ is cyclic. If $X(k)=\emptyset$, then $X\left(k_{v}\right)=\emptyset$ for infinitely many places $v$ of $k$. In particular $X$ satisfies the Hasse principle.

Proof. When $X$ is irreducible this is well-known, and follows, for example, from Lemma 2.2 and a theorem of Jordan [40, Thm. 4]. When $\Gamma$ is cyclic, the result is an easy application of Lemma 2.2 (if a generator of $\Gamma$ acts with a fixed point, then so does $\Gamma$ ).

Remark 2.4. For non-cyclic groups however, counter-examples to the Hasse principle always occur. Namely, let $k$ be a number field and let $\Gamma$ be a non-cyclic group which is realisable as a Galois group over $k$. Then it was shown in [46] 
and [47] that there exists some finite étale scheme over $k$ whose splitting field has Galois group $\Gamma$ and which is a counter-example to the Hasse principle.

In the solvable case, these results will suffice for our purposes. In the nonsolvable case however, Sonn's construction does not work for us since the schemes produced have very large degree in general. We therefore take a different approach, using work of Kedlaya [23].

We next give a condition for the failure of the Hasse principle in terms of the associated étale algebra.

Lemma 2.5. Let $K_{1}, \ldots, K_{r}$ be number fields which contain, yet are strictly larger than, a fixed number field $k$. Let

$$
X=\operatorname{Spec} K_{1} \sqcup \cdots \sqcup \operatorname{Spec} K_{r},
$$

considered as a scheme over $k$. Then $X$ fails the Hasse principle if and only if for each prime ideal $\mathfrak{p}$ of $k$, one of the fields $K_{i}$ admits an unramified prime ideal above $\mathfrak{p}$ of inertia degree 1 .

Proof. By construction $X$ admits no $k$-point. The key relevant fact for the proof is that if $F / E$ is an unramified extension of non-archimedean local fields of inertia degree 1, then $F=E$ (see [37, §III.5]). This easily implies that $X$ is soluble at each non-archimedean place of $k$ if and only if for each prime ideal $\mathfrak{p}$ of $k$, one of the fields $K_{i}$ admits an unramified prime ideal above $\mathfrak{p}$ of inertia degree 1 . It follows from Lemma 2.2 however that $X$ is soluble at each non-archimedean place of $k$ if and only if it is soluble at all places of $k$, and the result follows.

Remark 2.6. M. Stoll [49, Prop. 5.12] has shown that the finite abelian descent obstruction is the only one to the Hasse principle for finite étale schemes. It is also quite simple to see that the Brauer-Manin obstruction is the only one to the Hasse principle in this case. Indeed, let $X$ be a finite étale scheme over $k$ which fails the Hasse principle and let $\left(x_{v}\right) \in X\left(\mathbb{A}_{k}\right)$ be an adelic point. Choose an irreducible component $X_{0}$ of $X$ for which the set

$$
S=\left\{v \in \operatorname{Val}(k): x_{v} \in X_{0}\left(k_{v}\right)\right\},
$$

contains a non-complex place $v_{0}$. Note that the complement of $S$ in $\operatorname{Val}(k)$ is infinite, by Lemma 2.3. Write $X_{0}=\operatorname{Spec} K$, so that $x_{v_{0}}$ corresponds to some unramified place $w_{0} \mid v_{0}$ of $K$ of inertia degree 1 . Then by class field theory, there exists $b_{0} \in \operatorname{Br} X_{0}=\operatorname{Br} K$ such that $\operatorname{inv}_{w_{0}} b_{0} \neq 0$, but such that $\operatorname{inv}_{w} b_{0}=0$ for all other $w \in \operatorname{Val}(K)$ above the places of $S$. Hence the adelic point $\left(x_{v}\right)$ is not orthogonal to the Brauer group element

$$
b=\left(b_{0}, 0\right) \quad \in \operatorname{Br} X_{0} \oplus \operatorname{Br}\left(X \backslash X_{0}\right)=\operatorname{Br} X .
$$

Thus $X\left(\mathbb{A}_{k}\right)^{\mathrm{Br}}=\emptyset$, as required.

2.1.3. Existence of counter-examples. We finish by gathering some results on the existence of finite étale schemes which fail the Hasse principle. The first result says that in the solvable case, we may choose our field extensions such that the solubility conditions at the ramified primes in Lemma 2.2 are always satisfied.

Lemma 2.7. Let $k$ be a number field and let $\Gamma$ be a solvable group. Then there exists a Galois extension $K / k$ with Galois group $\Gamma$ with the following property: 
Let $X$ be a finite étale $k$-scheme with splitting field $K$, such that each conjugacy class of $\Gamma$ acts with a fixed point on the associated $\Gamma$-set $X(K)$, but $\Gamma$ acts without a fixed point. Then $X$ is a counter-example to the Hasse principle.

Proof. It is shown in the proof of [46, Thm. 2] that there exists a finite Galois extension $K / k$ with Galois group $\Gamma$, all of whose decomposition groups are cyclic. The result then follows from Lemma 2.1 and Lemma 2.2.

We also construct explicit counter-examples when $\Gamma=\mathbb{Z} / 2 \mathbb{Z} \times \mathbb{Z} / 2 \mathbb{Z}$.

Lemma 2.8. Let $k$ be a number field. Then there exist $a, b \in k^{*}$ such that the $k$-scheme

$$
\left(x^{2}-a\right)\left(x^{2}-b\right)\left(x^{2}-a b\right)=0,
$$

fails the Hasse principle.

Proof. Choose rational primes $p$ and $q$ such that $p \equiv 1(\bmod 8), q \equiv 1(\bmod p)$ and

$$
\mathbb{Q}(\sqrt{p}), \mathbb{Q}(\sqrt{q}), \mathbb{Q}(\sqrt{p q}) \not \subset k .
$$

This is possible since there are infinitely many such primes by Dirichlet's theorem, and since $k$ contains only finitely many subfields by Galois theory. A simple computation shows that the scheme

$$
\left(x^{2}-p\right)\left(x^{2}-q\right)\left(x^{2}-p q\right)=0,
$$

over $\mathbb{Q}$ is a counter-example to the Hasse principle, and that it also remains a counter-example to the Hasse principle upon base-change to $k$.

2.2. Del Pezzo surfaces. In this section we gather various facts we shall require about the geometry of del Pezzo surfaces. For background on del Pezzo surfaces, we use [31], [14, Ch. 8] and [25, III.3].

\subsubsection{Classification.}

Definition 2.9. Let $k$ be a field. A del Pezzo surface over $k$ is a smooth projective surface $S$ over $k$ with ample anticanonical divisor $\left(-K_{S}\right)$. The degree $d$ of $S$ is defined to be the self-intersection number $d=\left(-K_{S}\right)^{2}$.

The degree $d$ of any del Pezzo surface $S$ satisfies $1 \leq d \leq 9$. If $k$ is algebraically closed, any del Pezzo surface is isomorphic to either $\mathbb{P}^{2}, \mathbb{P}^{1} \times \mathbb{P}^{1}$, or the blow-up of $\mathbb{P}^{2}$ in $9-d$ points in general position $(d \leq 8)$. Here by general position, we mean that no three lie on a line, no six lie on a conic, and no eight lie on a cubic which is singular at one of the points. In which case, the exceptional curves on $S$ consist of the exceptional curves of the blow-ups of the points, together with the strict transforms of the following curves (see [31, Thm. 26.2]).

(1) Lines through two of the points.

(2) Conics through five of the points.

(3) Cubic curves through seven of the points, with a double point at exactly one of them.

(4) Quartic curves through eight of the points, with a double point at exactly three of them.

(5) Quintic curves through eight of the points, with a double point at exactly six of them. 
(6) Sextic curves through eight of the points, with a triple point at exactly one of them and double points at the rest.

For general $k$, one may construct del Pezzo surfaces by blowing-up collections of closed points of $\mathbb{P}^{2}$ in general position. Here we say that a collection of closed points is in general position if the corresponding points over $\bar{k}$ are in general position. Note that, in general, not every del Pezzo surface arises in this way, as surfaces constructed in this manner will always be non-minimal.

2.2.2. The lines. Much of the arithmetic and geometry of del Pezzo surfaces is governed by the lines on the surface. We now let $k$ be a perfect field.

Definition 2.10. Let $S$ be a del Pezzo surface of degree $d$ over $k$ and let $L$ be a curve on $S$ defined over $k$. Then we call $L$ a line if:

(1) $L \cdot\left(-K_{S}\right)=3$, if $d=9$.

(2) $L \cdot\left(-K_{S}\right)=2$, if $S_{\bar{k}} \cong \mathbb{P}^{1} \times \mathbb{P}^{1}$.

(3) $L \cdot\left(-K_{S}\right)=1$ and $p_{a}(L)=0$, otherwise.

When $d \leq 7$ such curves are exactly the $(-1)$-curves on $S$. In the other cases we have chosen a definition which reflects the geometry over the algebraic closure. Indeed, a curve $L$ in $\mathbb{P}^{2}$ with $L \cdot\left(-K_{\mathbb{P}^{2}}\right)=3$ is exactly a line in the usual sense (since $-K_{\mathbb{P}^{2}}=3 L$ ), and moreover a curve $L$ in $\mathbb{P}^{1} \times \mathbb{P}^{1}$ with $L \cdot\left(-K_{\mathbb{P}^{1} \times \mathbb{P}^{1}}\right)=2$ is also exactly a line in the usual sense, once one embeds $\mathbb{P}^{1} \times \mathbb{P}^{1}$ into $\mathbb{P}^{3}$ as a quadric surface via the Segre embedding. Note that del Pezzo surfaces of degree 1 contain curves $C$ such that $C \cdot\left(-K_{S}\right)=1$ but such that $C$ is not a line; any curve in the anticanonical linear system is such a curve.

For a del Pezzo surface $S$, we shall denote by $\mathcal{L}(S)$ the Hilbert scheme of lines on $S$. This is the scheme which parametrises the lines inside $S$, whose existence is a special case of a general result of Grothendieck [18, Exposé 221, Thm. 3.1].

Proposition 2.11. Let $S$ be a del Pezzo surface of degree d over $k$. Then $\mathcal{L}(S)$ is a reduced projective scheme over $k$, which satisfies the following:

(1) $\mathcal{L}(S)_{\bar{k}} \cong \mathbb{P}^{2}$, if $d=9$.

(2) $\mathcal{L}(S)_{\bar{k}}=\mathbb{P}^{1} \sqcup \mathbb{P}^{1}$, if $S_{\bar{k}} \cong \mathbb{P}^{1} \times \mathbb{P}^{1}$.

(3) $\mathcal{L}(S)$ is a finite étale scheme of degree

\begin{tabular}{c|c|c|c|c|c|c|c|c}
$d$ & 8 & 7 & 6 & 5 & 4 & 3 & 2 & 1 \\
\hline $\operatorname{deg} \mathcal{L}(S)$ & 1 & 3 & 6 & 10 & 16 & 27 & 56 & 240
\end{tabular}

otherwise.

Proof. The fact that $\mathcal{L}(S)$ is reduced is well-known, see for instance [42, Prop. 3.5]. As the formation of the Hilbert scheme $\mathcal{L}(S)$ respects base-change, we may assume that $k$ is algebraically closed. In which case, (11) follows from the classical duality of lines in the projective plane, and (2) follows from the fact that $\mathbb{P}^{1} \times \mathbb{P}^{1}$ admits exactly two distinct rulings by lines. For (3), the calculation of the degree of $\mathcal{L}(S)$ follows from the calculation of the number of lines on del Pezzo surfaces over algebraically closed fields [31, Cor. 25.5.4]. 
We shall say that a del Pezzo surface $S$ of degree $d \leq 7$ over $k$ is split if $\mathcal{L}(S)$ is split (as a finite étale scheme). There is a unique smallest Galois extension over which $S$ becomes split, called the splitting field of $S$.

We shall say that a class of del Pezzo surfaces over a number field $k$ satisfies the Hasse principle for lines, if for each surface $S$ in the class, the Hilbert scheme $\mathcal{L}(S)$ satisfies the Hasse principle.

2.2.3. Graphs of lines. Let $d \leq 7$ and let $S$ be a del Pezzo surface of degree $d$ over an algebraically closed field $k$. Denote by $G(S)$ the graph of lines of $S$ (see [31, §26.9]). This has one vertex for each line on $S$, and given two distinct lines $L_{1}$ and $L_{2}$ on $S$, there is an edge of multiplicity $L_{1} \cdot L_{2}$ between the corresponding vertices. Note that multiple edges occur if and only if $d \leq 2$.

There exists a graph $G_{d}$ such that if $S$ is a del Pezzo surface of degree $d$, then $G(S) \cong G_{d}$. This graph may be defined via the root system $\mathbf{E}_{9-d}$ (see [14, §8.2.2]). Namely, there is one vertex for each lattice point $\ell \in \mathbf{E}_{9-d}$ which satisfies $(\ell, \ell)=-1$, and given two distinct such points $\ell_{1}$ and $\ell_{2}$, there is an edge of multiplicity $\left(\ell_{1}, \ell_{2}\right)$ between the corresponding vertices.

We define the automorphism group $\operatorname{Aut}\left(G_{d}\right)$ of $G_{d}$ to be those automorphisms of the vertex set of $G_{d}$ which preserve edge multiplicities between vertices (in particular we are not interested in "automorphisms" of $G_{d}$ which fix every vertex, yet permute multiple edges between adjacent vertices). The automorphism group of $G_{d}$ is the Weyl group $W\left(\mathbf{E}_{9-d}\right)$ [31, Thm. 23.8]. We have the following explicit descriptions.

- $G_{7}$ : Path graph on 3 vertices. $W\left(\mathbf{E}_{2}\right) \cong W\left(\mathbf{A}_{2}\right) \cong \mathbb{Z} / 2 \mathbb{Z}$.

- $G_{6}$ : Cycle graph on 6 vertices. $W\left(\mathbf{E}_{3}\right) \cong W\left(\mathbf{A}_{1} \times \mathbf{A}_{2}\right) \cong D_{6}$.

- $G_{5}$ : The Petersen graph. $W\left(\mathbf{E}_{4}\right) \cong W\left(\mathbf{A}_{4}\right) \cong S_{5}$.

- $G_{4}$ : The Clebsch graph. $W\left(\mathbf{E}_{5}\right) \cong W\left(\mathbf{D}_{5}\right) \cong(\mathbb{Z} / 2 \mathbb{Z})^{4} \rtimes S_{5}$.

- $G_{3}$ : The (dual of the) Schläfli graph. $W\left(\mathbf{E}_{6}\right)$.

Let now $S$ be a del Pezzo surface of degree $d$ over a perfect field $k$ with splitting field $K$ and $\Gamma=\operatorname{Gal}(K / k)$. Here we define $G(S)=G\left(S_{\bar{k}}\right)$. The action of $\Gamma$ on the lines of $S_{\bar{k}}$ induces an action of $\Gamma$ on $G(S)$. The vertices therefore form a $\Gamma$-set, whose associated finite étale scheme is exactly the Hilbert scheme $\mathcal{L}(S)$ of lines of $S$. Choosing a graph isomorphism $G(S) \cong G_{d}$, we obtain an action of $\Gamma$ on $G_{d}$, which identifies $\Gamma$ with a subgroup of $\operatorname{Aut}\left(G_{d}\right)=W\left(\mathbf{E}_{9-d}\right)$. Different choices of the graph isomorphism give rise to conjugate subgroups, so we often view this as an action of $\Gamma$ on $G_{d}$, well-defined up to conjugacy. If $O_{1}, \ldots, O_{r}$ denote the orbits of the action of $\Gamma$ on the vertices of $G(S)$, we call the unordered list $\left[\# O_{1}, \ldots, \# O_{r}\right]$ the orbit type of $S$.

\section{The Hasse Principle for lines on del Pezzo Surfaces}

The aim of this section is to prove Theorem 1.4.

3.1. Proof strategy. The cases $d=8$ and $d=9$ shall be handled separately, using basic geometric and arithmetic facts, together with Proposition 2.11. For $d \leq 7$, the following proposition is the key of our strategy.

Proposition 3.1. Let $S$ be a del Pezzo surface of degree $d \leq 7$ over a number field $k$. Let $K / k$ be the splitting field of $S$ with Galois group $\Gamma$. Then $S$ contains 
a line over almost all completions of $k$, but no line over $k$, if and only if each conjugacy class of $\Gamma$ acts with a fixed point on the graph $G_{d}$, but $\Gamma$ acts without a fixed point on $G_{d}$.

In which case, for every place $v$ of $k$ which is either archimedean or unramified in $K$, the surface $S$ admits a line over $k_{v}$.

Proof. This follows immediately from Lemma 2.2 .

Using the criterion given by Proposition 3.1, the first step is purely computational. Namely for each $d \leq 7$, we enumerate all conjugacy classes of subgroups of $\operatorname{Aut}\left(G_{d}\right)=W\left(\mathbf{E}_{9-d}\right)$. We store those subgroups $\Gamma$ which have no fixed point when acting on $G_{d}$, yet are such that each conjugacy class of $\Gamma$ admits a fixed point. This gives a list for the Galois groups of the splitting fields of del Pezzo surfaces which could fail the Hasse principle for lines, together with the associated Galois actions on the lines of the surface. Note that by Lemma 2.3, such subgroups must be non-transitive and non-cyclic. This classification can be done easily by hand for $d=7$ and 6 , but such computations become increasingly more difficult, so for $d \leq 5$ we use a computer.

Fix now some $d \leq 7$ and suppose that we have found such a subgroup $\Gamma$ acting on $G_{d}$. For each field extension $K / k$ with Galois group $\Gamma$, we may associate via our dictionary a finite étale $k$-scheme $X$. Note that by construction, this scheme will be soluble at all those places of $k$ which are either archimedean or unramified in $K$. The first step is to find such a field $K$ for which the associated scheme $X$ fails the Hasse principle, by taking care of the conditions at the ramified places. We do this using the results obtained in \$2.1.2 and \$2.1.3. In the most difficult case of $S_{5}$, we also require work of Kedlaya [23].

The next step is the "inverse Galois problem" for del Pezzo surfaces, namely to find a del Pezzo surface $S$ whose Hilbert scheme of lines is isomorphic to the scheme $X$ found above. We are fortunate since when $d \geq 3$, the cases which arise correspond to rational surfaces. We therefore construct them by blow-ups of $\mathbb{P}^{2}$ followed by certain blow-downs. Only when $d=2$ or 1 do non-rational examples occur, and here we construct counter-examples using conic bundles.

3.1.1. Implementing the strategy. In the interests of clarity, we now explain how to construct the graphs $G_{d}$, together with the associated action of $W\left(\mathbf{E}_{9-d}\right)$, and how one runs the computational part of the proof strategy in Magma. We do this now in the case of the graph $G_{2}$, explaining briefly at the end what changes need to be made to construct the other graphs. The following commands

R_E7 := RootDatum("E7");

Cox_E7 := CoxeterGroup(R_E7);

W_E7 := StandardActionGroup (Cox_E7);

construct the group $W\left(\mathbf{E}_{7}\right)$ viewed as a permutation group on 56 letters, corresponding to the 56 vertices of the graph $G_{2}$. We now enumerate the conjugacy classes of subgroups of $W\left(\mathbf{E}_{7}\right)$ on using the command

SubgroupClasses (W_E7);

and find those subgroups $\Gamma \subseteq W\left(\mathbf{E}_{7}\right)$ which satisfy the conditions of Proposition 3.1, using standard commands in Magma for computing with permutation groups. 
We construct the edges of $G_{2}$ by constructing the "intersection matrix" of $G_{2}$. This is defined in a similar manner to the usual adjacency matrix of the graph, except that we define the intersection of a vertex with itself to be $(-1)$, rather than 0 , as these correspond to $(-1)$-curves. We first consider the action of $W\left(\mathbf{E}_{7}\right)$ on unordered pairs of distinct elements of the set $\{1, \ldots, 56\}$, via the command subs2 := Subsets (Set (GSet (W_E7)), 2);

The action of $W\left(\mathbf{E}_{7}\right)$ on subs 2 decomposes into 3 orbits, corresponding to pairs of vertices with edges between them of multiplicities 0,1 and 2 . The orbits have sizes 28,756 and 756 . The smallest orbit corresponds to those pairs of vertices which have an edge between them of multiplicity 2 . To identify which orbit of size 756 corresponds to the adjacent vertices, one considers both possible options in constructing the intersection matrix of $G_{2}$. One then notices that only one possibility leads to an intersection matrix with the required rank 8 , the other giving a matrix of rank 29.

To construct the graphs $G_{3}$ and $G_{1}$, one applies a similar method, but using the root systems $W\left(\mathbf{E}_{6}\right)$ and $W\left(\mathbf{E}_{8}\right)$, respectively. The StandardActionGroup command in Magma does not give the required group actions for $G_{d}$ when $d \geq 4$. However these groups, together with their required actions, are part of Magma's library of transitive groups. The commands

W_E3 := TransitiveGroup $(6,3)$;

W_E4 := TransitiveGroup $(10,13)$;

W_E5 := TransitiveGroup $(16,1328)$;

construct the corresponding groups for $G_{6}, G_{5}$ and $G_{4}$ respectively (the case $G_{7}$ in the proof being trivial). These programs can be found on the authors' webpages.

Remark 3.2. The part of the algorithm which dominates the run time is the step which involves enumerating the conjugacy classes of subgroups of $W\left(\mathbf{E}_{9-d}\right)$. For $d \geq 2$ this takes only a matter of seconds on a modern desktop computer, however the case $d=1$ takes around 30 minutes.

We now proceed with the proof of Theorem 1.4, following this strategy.

3.2. Degree 9. Let $S$ be a del Pezzo surface of degree 9. By Proposition 2.11, the scheme $\mathcal{L}(S)$ is also a del Pezzo surface of degree 9 . Therefore, by a classical theorem of Châtelet (see e.g. [10, Cor. 2.6]), the scheme $\mathcal{L}(S)$ satisfies the Hasse principle, as required.

3.3. Degree 8. If $S_{\bar{k}} \neq \mathbb{P}^{1} \times \mathbb{P}^{1}$ then $S_{\bar{k}}$ contains only a single line, hence $S$ contains a line over $k$. Thus the Hasse principle for lines trivially holds.

Let now $S$ be a twist of $\mathbb{P}^{1} \times \mathbb{P}^{1}$. Fix a choice of isomorphism Pic $S_{\bar{k}} \cong \mathbb{Z}^{2}$, to obtain an injective map Pic $S \rightarrow \mathbb{Z}^{2}$. We say that a divisor $D$ on $S$ has type $(a, b)$, if its class has image $(a, b)$ under this map. The lines on $S$ are exactly the effective divisors of type $(1,0)$ or $(0,1)$.

Lemma 3.3. Let $S$ be a twist of $\mathbb{P}^{1} \times \mathbb{P}^{1}$ over a number field $k$. Suppose that $S$ contains a line over all but finitely many completions of $k$. Then $\mathcal{L}(S)$ is reducible. In particular, there exist conics $C_{1}$ and $C_{2}$ over $k$, unique up to isomorphism and reordering, such that

$$
S \cong C_{1} \times C_{2} \quad \text { and } \quad \mathcal{L}(S) \cong C_{1} \sqcup C_{2} .
$$


Proof. By Proposition 2.11, the scheme $\pi_{0}(\mathcal{L}(S)$ ) (see \$1.1) is a finite étale scheme of degree 2. By assumption, it has a point over all but finitely many completions of $k$. It follows easily from Lemma 2.2 that $\pi_{0}(\mathcal{L}(S))$ is split. Hence, by Proposition 2.11 there exist conics $C_{1}$ and $C_{2}$ such that

$$
\mathcal{L}(S) \cong C_{1} \sqcup C_{2} .
$$

In particular, the surface $S$ contains divisors of type $(2,0)$ and $(0,2)$. The morphisms associated to these divisors give maps $S \rightarrow C_{i}$, which when combined together give the required isomorphism $S \cong C_{1} \times C_{2}$.

It is quite easy to construct counter-examples here. Namely, by the classification of conics over $k$ (see e.g [35, Thm. 72.1]), there exist two conics $C_{1}$ and $C_{2}$ over $k$ with $C_{1}(k)=\emptyset$ and $C_{2}(k)=\emptyset$, yet for each place $v$ of $k$ there is some $i \in\{1,2\}$ such that $C_{i}\left(k_{v}\right) \neq \emptyset$. In which case, the surface

$$
S=C_{1} \times C_{2},
$$

is a counter-example to the Hasse principle for lines. Indeed, the scheme $\mathcal{L}(S)=$ $C_{1} \sqcup C_{2}$ fails the Hasse principle. On the other hand, we have the following.

Lemma 3.4. Let $S \subset \mathbb{P}^{3}$ be a smooth quadric surface over a number field $k$. Then $S$ satisfies the Hasse principle for lines.

Proof. Assume that $S$ contains a line over every completion of $k$. Then $S$ is everywhere locally soluble, hence Pic $S \cong\left(\operatorname{Pic} S_{\bar{k}}\right)^{G_{k}}$ (see e.g. [10, Cor. 2.5]). By Lemma 3.3 we know that $S$ contains a divisor of type $(2,0)$. Thus any divisor class on $S_{\bar{k}}$ of type $(1,0)$ is Galois invariant, hence is defined over $k$. Therefore $S$ contains a line, so the Hasse principle for lines holds.

3.4. Degree 7. The graph $G_{7}$ is the path graph on 3 vertices. In particular the automorphism group of the graph fixes a vertex, so there is always a line defined over $k$. Hence the Hasse principle for lines trivially holds.

3.5. Degree 6. Here the graph $G_{6}$ is the cycle graph on 6 vertices, which we identify with a regular hexagon. The automorphism group is the dihedral group $D_{6}$, acting in the usual way.

Let $\Gamma \subseteq D_{6}$ be a subgroup. If $\Gamma$ is transitive or cyclic, then by Lemma 2.3 the Hasse principle holds. The group $D_{6}$ contains only two conjugacy classes of non-transitive non-cyclic subgroups, which are isomorphic to the Klein four group $V_{4}$ and the dihedral group $D_{3}$ respectively. Both these groups admit an element which acts as a non-trivial rotation, hence has no fixed point. Thus by Proposition 3.1, the Hasse principle for lines holds.

\subsection{Degree 5 .}

3.6.1. Results of computer experiments. We now present the results obtained from our computer experiments, following our proof strategy. Here $G_{5}$ is the Petersen graph, with automorphism group $W\left(\mathbf{E}_{4}\right) \cong W\left(\mathbf{A}_{4}\right) \cong S_{5}$ of order 120 . Our results are summarised in Table 1.

We now explain the notation in the table, with similar notation occurring for the other tables in our paper. The first column gives the number of conjugacy classes of subgroups of $W\left(\mathbf{E}_{4}\right)$. We only consider subgroups up to conjugacy, as 


\begin{tabular}{c|c|c|c|c} 
subgroups & transitive & cyclic & fixed point & satisfy Prop. 3.1 \\
\hline 19 & 3 & 7 & 9 & 2
\end{tabular}

TABle 1. Results of experiments for del Pezzo surfaces of degree 5

conjugate actions give rise to isomorphic finite étale schemes. We then list the number of these which act transitively on $G_{5}$, the number which are cyclic, the number which act with a fixed point, and the number which satisfy the conditions of Proposition 3.1. Ultimately only the last column will be of interest, but we have included the other columns to give a more complete picture.

The two subgroups of $W\left(\mathbf{E}_{4}\right)$ which satisfy Proposition 3.1 are isomorphic to $V_{4}$ and $A_{4}$, respectively. They have the following orbit types.

(1) $V_{4}$ with orbit type $[2,2,2,4]$.

(2) $A_{4}$ with orbit type $[4,6]$.

In both cases, the orbits of length 4 consist of mutually non-adjacent vertices. This means that the corresponding del Pezzo surfaces (if they exist) contain 4 skew lines. Contracting these lines, we obtain a del Pezzo surface $S^{\prime}$ of degree 9. A theorem of Enriques and Swinnerton-Dyer (see e.g. [44, Cor. 3.1.5]) states that any del Pezzo surface of degree 5 contains a rational point, in particular so does $S^{\prime}$ and hence $S^{\prime} \cong \mathbb{P}^{2}$. Thus any such surface is obtained by blowing-up $\mathbb{P}^{2}$ in a closed point of degree 4 . We now explain how to realise these orbit types and how to construct counter-examples in each case.

3.6.2. $V_{4}$. Let $K / k$ be a $V_{4}$-extension and let $S$ be the blow-up of $\mathbb{P}^{2}$ in a closed point of degree 4 in general position, with residue field $K$. Using the description of the lines on $S$ given in $\$ 2.2 .1$, it is simple to see that $S$ has the required orbit type $[2,2,2,4]$. In particular, there are three non-conjugate subgroups of $V_{4}$ of order 2 which act with a fixed point on $G_{5}$. To see that $S$ satisfies the conditions of Proposition 3.1, it suffices to note the following:

- $V_{4}$ contains exactly three subgroups of order 2 .

- Every element of $V_{4}$ lies inside such a subgroup.

Therefore $S$ contains a line over almost all completions of $k$. To take care of the ramified primes, we may choose $K / k$ as in Lemma 2.7, which guarantees that $S$ contains lines over all completions of $k$. Thus $S$ is the required counter-example to the Hasse principle for lines.

As an example, one may blow-up the closed point which over $\bar{k}$ is the union of

$$
[\sqrt{a}: \sqrt{b}: 1],[-\sqrt{a}: \sqrt{b}: 1],[\sqrt{a}:-\sqrt{b}: 1] \text { and }[-\sqrt{a}:-\sqrt{b}: 1] \text {, }
$$

where $a, b$ are as in Lemma 2.8 ,

3.6.3. $A_{4}$. Here the construction is similar to the previous case. One takes $S$ to be the blow-up of $\mathbb{P}^{2}$ in a closed point of degree 4 , whose splitting field $K$ has Galois group $A_{4}$. The action of $A_{4}$ is transitive on the collection of lines passing through these 4 points over $\bar{k}$, thus the orbit type is the required $[4,6]$. In particular there are subgroups of $A_{4}$ of orders 2 and 3 which act with a fixed point on $G_{5}$. To see that $S$ satisfies the conditions of Proposition 3.1, it suffices to note the following:

- $A_{4}$ contains only one conjugacy class of subgroups of orders 2 and 3. 
- Every element of $A_{4}$ lies inside such a subgroup.

Thus on choosing $K$ as in Lemma 2.7, we obtain the required counter-example.

As an explicit example of $K$ which works when $k=\mathbb{Q}$, one may take the splitting field of the polynomial

$$
f(x)=x^{4}-x^{3}-7 x^{2}+2 x+9 .
$$

This polynomial was taken from [24]. Let $L$ be the field defined by $f$. It has discriminant $163^{2}$ and its Galois closure $K$ has Galois group $A_{4}$. It follows from Lemma 2.5 that this gives the required counter-example, on noticing that we have a factorisation

$$
(163)=\mathfrak{p}_{1} \mathfrak{p}_{2}^{3},
$$

into prime ideals of $L$, where both $\mathfrak{p}_{1}$ and $\mathfrak{p}_{2}$ have inertia degree 1 .

\subsection{Degree 4.}

3.7.1. Results of computer experiments. Here $G_{4}$ is the Clebsch graph, with automorphism group $W\left(\mathbf{E}_{5}\right) \cong W\left(\mathbf{D}_{5}\right) \cong(\mathbb{Z} / 2 \mathbb{Z})^{4} \rtimes S_{5}$ of order 1920. Our results are summarised in Table 2 .

\begin{tabular}{c|c|c|c|c} 
subgroups & transitive & cyclic & fixed point & satisfy Prop. 3.1 \\
\hline 197 & 51 & 18 & 19 & 0
\end{tabular}

TABle 2. Results of experiments for del Pezzo surfaces of degree 4

There are 116 conjugacy classes of subgroups of $W\left(\mathbf{E}_{5}\right)$ which are not transitive, nor cyclic, nor admit a fixed point. Moreover, none of these satisfy the conditions of Proposition 3.1. Hence the Hasse principle for lines holds. We will give a non-computer-assisted proof of this result in $\$ 5$.

\subsection{Degree 3.}

3.8.1. Results of computer experiments. Here $G_{3}$ is the (dual of the) Schläfli graph, with automorphism group $W\left(\mathbf{E}_{6}\right)$ of order 51840. Our results are summarised in Table 3 .

\begin{tabular}{c|c|c|c|c} 
subgroups & transitive & cyclic & fixed point & satisfy Prop. 3.1 \\
\hline 350 & 25 & 25 & 172 & 3
\end{tabular}

TABle 3. Results of experiments for del Pezzo surfaces of degree 3

Our experiments show that exactly 3 out of the 350 conjugacy classes of $W\left(\mathbf{E}_{6}\right)$ satisfy the conditions of Proposition 3.1. Surprisingly, these all admit a very special orbit type. The three subgroups which arise are as follows.

(1) $D_{5}$ with orbit type $[2,5,5,5,10]$.

(2) $\mathbb{Z} / 5 \mathbb{Z} \rtimes \mathbb{Z} / 4 \mathbb{Z}$ with orbit type $[2,5,10,10]$.

(3) $S_{5}$ with orbit type $[2,5,10,10]$.

Here $\mathbb{Z} / 5 \mathbb{Z} \rtimes \mathbb{Z} / 4 \mathbb{Z}$ denotes a semi-direct product of $\mathbb{Z} / 5 \mathbb{Z}$ with $\mathbb{Z} / 4 \mathbb{Z}$ for which the induced morphism $\mathbb{Z} / 4 \mathbb{Z} \rightarrow(\mathbb{Z} / 5 \mathbb{Z})^{*}$ is an isomorphism (such a group is unique up to isomorphism).

In each case, the orbit of length two consists of two non-adjacent vertices in the graph, and there is also an orbit of length five consisting of mutually nonadjacent vertices. Therefore any cubic surface of this type, if it exists, admits two 
skew lines defined over a quadratic field extension. Such surfaces are rational. Indeed, contracting the two skew conjugate lines we obtain a del Pezzo surface of degree 5, and any such surface is rational [31, Thm. 29.4].

3.8.2. Constructing these surfaces. We now explain how to construct these surfaces. Choose two closed points $P$ and $Q$ in $\mathbb{P}^{2}$ of degrees 2 and 5, respectively, such that $P \sqcup Q$ is in general position. The del Pezzo surface $S^{\prime}=\mathrm{Bl}_{P \sqcup Q} \mathbb{P}^{2}$ of degree 2 contains exactly two lines. These are the strict transforms $\widetilde{L}$ and $\widetilde{C}$ of the line $L$ through the two quadratic points and the conic $C$ passing through the five quintic points over $\bar{k}$. Contracting $\widetilde{L}$, we obtain a cubic surface $S$ which contains no lines, since $\widetilde{C}$ and $\widetilde{L}$ intersect. Note that if we chose instead to contract $\widetilde{C}$, we would obtain an isomorphic surface, as $\widetilde{L}$ and $\widetilde{C}$ are exchanged by the Geiser involution on $S^{\prime}$ (see [14, §8.7.2]). One way to see this is by noting that the Geiser involution preserves the intersection product and fixes no line, combined with the fact that $\widetilde{C}$ is the unique line on $S^{\prime}$ with $\widetilde{C} \cdot \widetilde{L}=2$. Using the classification given in $\$ 2.2 .1$, the lines on $S$ correspond to the following curves:

(1) The 2 singular cubic curves which pass through all 7 points and which have a double point at exactly one of the quadratic points.

(2) The 5 exceptional curves above the quintic points.

(3) The 10 conics passing through the two quadratic points and three of the five quintic points.

(4) The 10 lines passing through one of the quadratic points and one of the quintic points.

This description gives a simple geometric way to visualise the Galois action on the lines. Such surfaces always contain an orbit of length two and an orbit of length five, which each consist of skew lines, together with an orbit of length ten given by curves of the type (44). The union of this latter orbit with the orbit of length two is a Galois invariant double six on the surface (see [20, Rem. V.4.9.] for a definition of this configuration). The Galois action on the curves occurring in (3) is the same as the action of the Galois group of the splitting field $K$ of $Q$ on pairs of distinct $K$-points of $Q$. Of the five transitive conjugacy classes of subgroups $\Gamma$ of $S_{5}$, there are two orbits of length five exactly when $\Gamma \cong \mathbb{Z} / 5 \mathbb{Z}$ or $\Gamma \cong D_{5}$. Hence this constructs surfaces with orbit types $[2,5,5,5,10]$ and $[2,5,10,10]$. Moreover, it is not difficult to see that every such surface arises in this way.

We now specialise the residue fields of $P$ and $Q$ to obtain the cases which arose from our computer experiments. In what follows we keep the above notation.

3.8.3. $D_{5}$. Assume now that the residue fields of $P$ and $Q$ belong to the same $D_{5}$-extension $K / k$. The orbit type is $[2,5,5,5,10]$ in this case. Thus there are subgroups of $D_{5}$ with orders 2 and 5 which act with a fixed point on $G_{3}$. To see that $S$ satisfies the conditions of Proposition 3.1, it suffices to note the following:

- $D_{5}$ contains only one conjugacy class of subgroups of orders 2 and 5.

- Every element of $D_{5}$ lies inside such a subgroup.

Therefore we see that $S$ contains a line over almost all completions of $k$. To obtain the required counter-example over $k$, we choose $K$ as in Lemma 2.7. 
For an explicit example of such a $K$ when $k=\mathbb{Q}$, one may take the splitting field of the polynomial

$$
f(x)=x^{5}-2 x^{4}+2 x^{3}-x^{2}+1 .
$$

Using Magma, one finds that the field $L$ defined by $f$ has discriminant $47^{2}$, and its splitting field $K$ is a $D_{5}$-extension of $\mathbb{Q}$. It follows from Lemma 2.5 that this gives the required counter-example, on noticing that we have a factorisation

$$
(47)=\mathfrak{p}_{1} \mathfrak{p}_{2}^{2} \mathfrak{p}_{3}^{2},
$$

into prime ideals of $L$, where each prime ideal has inertia degree 1.

3.8.4. $\mathbb{Z} / 5 \mathbb{Z} \rtimes \mathbb{Z} / 4 \mathbb{Z}$. Here we let $\Gamma=\mathbb{Z} / 5 \mathbb{Z} \rtimes \mathbb{Z} / 4 \mathbb{Z}$. We assume that the splitting field $K / k$ of $Q$ is a $\Gamma$-extension of $k$, and that the residue field of $P$ is the unique quadratic subfield of $K$. In which case the orbit type is $[2,5,10,10]$, hence there are subgroups of $\Gamma$ of orders 4 and 10 which act with a fixed point on $G_{3}$. To see that $S$ satisfies the conditions of Proposition 3.1, it suffices to note the following:

- $\Gamma$ contains only one conjugacy class of subgroups of order 4 and 10.

- Every element of $\Gamma$ lies inside such a subgroup.

As before, we obtain a counter-example on choosing $K$ as in Lemma 2.7.

Here one can write down a simple family of such fields when $k=\mathbb{Q}$. Namely, let $p$ be a prime which is congruent to 1 modulo 25 (infinitely such primes exist by Dirichlet's theorem). We then take $K$ to be the splitting field of the polynomial

$$
f(x)=x^{5}-p .
$$

It is simple to check that this is a $\Gamma$-extension and that the unique quadratic subfield of $K$ is $\mathbb{Q}(\sqrt{5})$. Therefore $\mathcal{L}(S)$ contains a subscheme isomorphic to

$$
\left(x^{2}-5\right)\left(x^{5}-p\right)=0 .
$$

It suffices to show that this scheme fails the Hasse principle. It satisfies the conditions of Lemma 2.2, hence we need only look at the ramified primes, namely 5 and $p$. As $p \equiv 1(\bmod 5)$, the field $\mathbb{Q}(\sqrt{5})$ splits at $p$, thus $S$ contains a line over $\mathbb{Q}_{p}$. Moreover, the polynomial $f$ has a non-singular root modulo 25 , hence by Hensel's lemma it admits a root over $\mathbb{Q}_{5}$. We therefore deduce that $S$ fails the Hasse principle for lines, as required.

The explicit cubic surface (1.1) was of this type, with $p=101$. We shall explain how we wrote down the equation in this case in 3.8 .6 ,

3.8.5. $S_{5}$. We now assume that the splitting field $K / k$ of $Q$ is an $S_{5}$-extension of $k$, and that the residue field of $P$ is the unique quadratic subfield of $K$. The orbit type here is $[2,5,10,10]$, hence there are subgroups of $S_{5}$ of orders 12, 12, 24 and 60 which act with a fixed point on $G_{3}$. Using the explicit description of the lines on $S$ given in $\$ 3.8 .2$, one checks that the two subgroups of order 12 are non-conjugate in $S_{5}$. To see that $S$ satisfies the conditions of Proposition 3.1, it suffices to note the following:

- Up to conjugacy, $S_{5}$ contains exactly two subgroups of order 12 , one of order 24 and one of order 60 .

- Every element of $S_{5}$ lies inside such a subgroup. 
Therefore $S$ contains a line over almost all completions of $k$. To handle the ramified primes we will have to choose $K$ very carefully. Note that Lemma 2.7 does not apply as $S_{5}$ is non-solvable.

We begin by noticing that the only solvable subgroups of $S_{5}$ which do not act with a fixed point on $G_{3}$ are all isomorphic to $\mathbb{Z} / 5 \mathbb{Z} \rtimes \mathbb{Z} / 4 \mathbb{Z}$ (in fact every subgroup of $S_{5}$ of order 20 has this form). We need only consider solvable subgroups, since decomposition groups are always solvable [37, Cor. IV.2.5]. We shall therefore choose $K$ so that such groups do not occur as decomposition groups.

Lemma 3.5. Let $k$ be a number field. Then there exists an $S_{5}$-extension $K / k$, none of whose decomposition groups is isomorphic to $\mathbb{Z} / 5 \mathbb{Z} \rtimes \mathbb{Z} / 4 \mathbb{Z}$.

Proof. We shall prove the result by constructing suitably infinitely many such fields over $\mathbb{Q}$ using [23], then take a compositum with $k$.

Let $F / \mathbb{Q}$ be a quintic field with square-free discriminant, whose Galois closure $K$ has Galois group $S_{5}$. If $N$ denotes the unique quadratic subfield of $K$, then the extension $K / N$ is unramified (see [23]). It follows that if $\mathfrak{p}$ is a ramified prime ideal of $K / \mathbb{Q}$, then $\mathfrak{p}$ has ramification index 2. Hence the decomposition group of $\mathfrak{p}$ has a normal subgroup of order 2 . However $\mathbb{Z} / 5 \mathbb{Z} \rtimes \mathbb{Z} / 4 \mathbb{Z}$ contains no such subgroup, thus $K$ satisfies the conditions of the lemma.

We now handle the case of a general number field $k$. Choose a quintic field $F / \mathbb{Q}$ as above such that the discriminant $\Delta_{F / \mathbb{Q}}$ is coprime to $\Delta_{k / \mathbb{Q}}$. Such fields exist by [23, Cor. 1.3]. Let $L$ be the Galois closure of $F$ and let $N$ be the unique quadratic subfield of $L$. On embedding $k$ and $L$ in a common algebraic closure, we obtain the following commutative diagram of field extensions

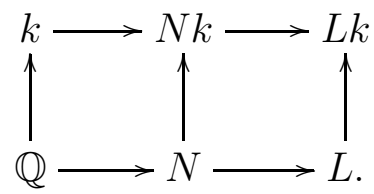

In order to prove the lemma with $K=L k$, we may apply a similar argument as above to reduce to showing that

(1) $k \subset L k$ is an $S_{5}$-extension.

(2) $N k \subset L k$ is unramified.

The assumption $\left(\Delta_{F / \mathbb{Q}}, \Delta_{k / \mathbb{Q}}\right)=1$ implies [33, Thm. 4.9] that $L$ and $k$ are linearly disjoint over $\mathbb{Q}$. Here (1) is then standard [27, Thm. VI.1.12]. Also as $N \subset L$ is unramified, (21) follows from [34, Prop. 7.2], as required.

Therefore choosing $K / k$ as in Lemma 3.5 and using Lemma 2.1, we see that the surface $S$ also admits a line over the completion of $k$ at every place which is ramified in $K$. Hence we obtain the required counter-example over $k$.

As an explicit choice over $\mathbb{Q}$, take $K$ to be the splitting field of the polynomial

$$
f(x)=x^{5}-x^{4}-5 x^{3}+5 x^{2}+2 x-1 .
$$

This polynomial was taken from [24]. The discriminant of the field defined by $f$ is equal to 101833 , which is prime, and the Galois group of $K$ is $S_{5}$. As the discriminant is square-free, the proof of Lemma 3.5 shows that it satisfies the required properties (in fact the decomposition group of each ramified prime ideal of $K$ is isomorphic to $\mathbb{Z} / 2 \mathbb{Z}$ here). 
3.8.6. An explicit counter-example. We now explain how we were able to write down the equation for the counter-example (1.1) given in the introduction. To do this, we shall use the constructions given in 33.8 .2 and $\$ 3.8 .4$.

Recall that we choose a point in $\mathbb{P}^{2}$ defined over $\mathbb{Q}(\sqrt[5]{101})$ and a point defined over $\mathbb{Q}(\sqrt{5})$ such that the associated orbits form seven points in general position, then blow them up to obtain an auxiliary del Pezzo surface $S^{\prime}$ of degree 2. The surface $S^{\prime}$ contains two exceptional curves that are $\mathbb{Q}$-rational, and blowing-down one of them we find a cubic surface $S$ with the desired properties.

To do this algorithmically, we determine three linearly independent cubic forms $c_{0}, c_{1}, c_{2}$ on $\mathbb{P}^{2}$ that vanish at the seven points. These define the anticanonical map $S^{\prime} \rightarrow \mathbb{P}^{2}$. We choose $c_{0}$ to be the product of a linear form vanishing on the size-two orbit and a quadratic form vanishing on the size-five orbit. We also determine a sextic form $s$ which admits a double point at each of the seven blowup points, and is not a linear combination of products of the cubic forms found. We then calculate the unique relation of the type

$$
s^{2}+f_{2}\left(c_{0}, c_{1}, c_{2}\right) s+f_{4}\left(c_{0}, c_{1}, c_{2}\right)=0
$$

between these forms. Here $f_{2}$ denotes a ternary quadratic form, while $f_{4}$ denotes a ternary quartic form.

Up to now, these computations are pure linear algebra. They lead to a del Pezzo surface of degree 2 with the explicit equation

$$
w^{2}+f_{2}\left(x_{0}, x_{1}, x_{2}\right) w+f_{4}\left(x_{0}, x_{1}, x_{2}\right)=0 \quad \subset \mathbb{P}(1,1,1,2) .
$$

By our choice of $c_{0}$, the pull-back of the line $x_{0}=0$, via the natural map $S^{\prime} \rightarrow \mathbb{P}^{2}$, decomposes into a union of two lines on $S^{\prime}$. Thus a linear transformation yields an equation of the form

$$
w^{2}+f_{2}^{\prime}\left(x_{0}, x_{1}, x_{2}\right) w+x_{0} \cdot f_{3}\left(x_{0}, x_{1}, x_{2}\right)=0,
$$

where $f_{2}^{\prime}$ is a ternary quadratic form and $f_{3}$ is a ternary cubic form. It is now classically known how to explicitly contract one the exceptional curves lying over the line $x_{0}=0$. As was shown by C. F. Geiser [19, §III] already in 1869, the result is the cubic surface $S$ given by the equation

$$
x_{0} \cdot w^{2}+f_{2}^{\prime}\left(x_{0}, x_{1}, x_{2}\right) w+f_{3}\left(x_{0}, x_{1}, x_{2}\right)=0 \quad \subset \mathbb{P}^{3} .
$$

This is the equation of the required counter-example. To obtain small coefficients as in (1.1), one may apply Kollar's minimisation algorithm [26, Prop. 6.4.2].

Remark 3.6. i) Explicit examples for the cases treated in 3.8 .3 and $\$ 3.8 .5$ may be obtained in a similar manner.

ii) As we have seen, surfaces with orbit type $[2,5,5,5,10]$ or $[2,5,10,10]$ have a Galois invariant double-six. Thus, the methods developed in [16] apply and lead to explicit equations for each of the three groups over $\mathbb{Q}$. Our approach here is simpler however, since it is more direct and easier to implement (for example, we do not need to perform any explicit Galois descent, as in [16]).

\subsection{Degree 2 .}




\begin{tabular}{c|c|c|c|c} 
subgroups & transitive & cyclic & fixed point & satisfy Prop. 3.1 \\
\hline 8074 & 32 & 60 & 350 & 60
\end{tabular}

TABLE 4. Results of experiments for del Pezzo surfaces of degree 2

3.9.1. Results of computer experiments. Here the group $\operatorname{Aut}\left(G_{2}\right)=W\left(\mathbf{E}_{7}\right)$ has order 2903040. Our results are summarised in Table 4.

Here a much wider range of behaviour occurs than in higher degrees. We therefore construct counter-examples in some special cases to illustrate this range, including some surfaces which are minimal.

Remark 3.7. Of the 60 conjugacy classes of subgroups which arise, exactly 2 are non-solvable, being isomorphic to $A_{5}$ and $A_{5} \times \mathbb{Z} / 2 \mathbb{Z}$, respectively. Moreover surfaces with these Galois actions are rational, being blow-ups of $\mathbb{P}^{1} \times \mathbb{P}^{1}$ in a closed point of degree six. It seems likely that the method used for cubic surfaces (\$3.8.2 and Lemma 3.5) can be adapted to construct counter-examples in such cases. Also, of these 60 conjugacy classes of subgroups, exactly 30 correspond to minimal surfaces. We shall not pursue all these additional cases in this paper.

\subsubsection{Some rational counter-examples.}

Lemma 3.8. Let $P, Q$ be closed points of $\mathbb{P}^{2}$ of degrees 3 and 4 , respectively, such that $P \sqcup Q$ lies in general position. Then $\mathrm{Bl}_{P \sqcup Q} \mathbb{P}^{2}$ contains no line.

Proof. It suffices to show that none of the curves in $\mathbb{P}^{2}$ described in $\$ 2.2 .1$ is Galois invariant. As there is no orbit of length 2 or 5 , no line nor conic is fixed by the Galois action. No cubic curve passing through the seven points which admits a double point at exactly one of them is Galois invariant, as there is no rational point being blown-up. This completes the proof.

Lemma 3.9. Let $P, Q$ be closed points of $\mathbb{P}^{2}$ of degrees 3 and 4 , respectively, such that $P \sqcup Q$ lies in general position. Assume that the splitting field $K$ of $Q$ is a $V_{4}$-or $A_{4}$-extension of $k$ which satisfies the conditions of Lemma 2.7. Then $\mathrm{Bl}_{P \sqcup Q} \mathbb{P}^{2}$ is a del Pezzo surface of degree 2 which is a counter-example to the Hasse principle for lines.

Proof. It follows from Lemma 3.8 that $S=\mathrm{Bl}_{P \sqcup Q} \mathbb{P}^{2}$ contains no line. However $S$ is the blow-up in a closed point of degree 3 of a del Pezzo surface of degree 5 which is a counter-example to the Hasse principle for lines (see 3.6 .2 and $\$ 3.6 .3$ ). In particular $S$ itself is a counter-example to the Hasse principle for lines.

Remark 3.10. One can check that the construction given in Lemma 3.9 yields 11 out of the 60 subgroups of $W\left(\mathbf{E}_{7}\right)$ which satisfy Proposition 3.1. Generically we obtain surfaces with orbit type

$$
[3,3,3,3,4,4,6,6,12,12]
$$

and all other surfaces thus obtained have refinements of this orbit type.

3.9.3. A minimal counter-example. We now give a minimal surface which is a counter-example to the Hasse principle for lines. We do this by constructing del Pezzo surfaces of degree 2 equipped with a conic bundle structure, using the methods of $[8, \S 5]$. Consider a conic bundle surface of the shape

$$
f(t) x^{2}+g(t) y^{2}+h(t) z^{2}=0 \quad \subset \mathbb{A}^{1} \times \mathbb{P}^{2},
$$


where

$$
f(t)=a(t-13)(2-t), \quad g(t)=b(t+14)(3-t), \quad h(t)=(t+2)(t-11),
$$

and $a, b \in k^{*}$. By [8, Prop. 5.2], the closure of this in $\mathbb{P}^{1} \times \mathbb{P}^{2}$ is a del Pezzo surface $S$ of degree 2. The natural projection realises $S$ as a conic bundle over $\mathbb{P}^{1}$, with exactly 6 singular fibres. If $a, b$ and $a b$ are not squares in $k$, then one checks that each of these singular fibres is non-split, and that exactly 2 fibres become split over each of one of the field extensions

$$
k(\sqrt{a}), k(\sqrt{b}), k(\sqrt{a b}) .
$$

Thus this conic bundle is relatively minimal, hence minimal by a result of Iskovskih [22, Thm. 4]. In particular the surface $S$ contains no line. Another result of Iskovskih implies that such surfaces are non-rational [21, Cor. 1.7]. If we choose $a, b$ as in Lemma 2.8, then for each place $v$ of $k$, at least one of the singular fibres splits into a union of lines. In particular, the surface contains a line over every completion of $k$, thus yields a counter-example to the Hasse principle for lines. Such surfaces are split over $k(\sqrt{a}, \sqrt{b})$, and have orbit type

$$
[2,2,2,2,2,2,2,2,2,2,2,2,4,4,4,4,4,4,4,4] \text {. }
$$

\subsection{Degree 1.}

3.10.1. Results of computer experiments. Here the group $\operatorname{Aut}\left(G_{1}\right)=W\left(\mathbf{E}_{8}\right)$ has order 696729600. Our results are summarised in Table 5 .

\begin{tabular}{c|c|c|c|c} 
subgroups & transitive & cyclic & fixed point & satisfy Prop. 3.1 \\
\hline 62092 & 60 & 112 & 7735 & 8742
\end{tabular}

TABle 5. Results of experiments for del Pezzo surfaces of degree 1

The number of possible subgroups is so large that it is not feasible with current technology and methods to consider all cases. Therefore, as before, we simply construct counter-examples in some special cases. We remark that out of the 8742 conjugacy classes of subgroups of $W\left(\mathbf{E}_{8}\right)$ which satisfy the conditions of Proposition 3.1, exactly 7775 are solvable.

\subsubsection{Some rational counter-examples.}

Lemma 3.11. Let $P, Q$ be closed points of $\mathbb{P}^{2}$ of degree 4 such that $P \sqcup Q$ lies in general position. Then $\mathrm{Bl}_{P \sqcup Q} \mathbb{P}^{2}$ contains no line.

Proof. As in Lemma 3.8, an inspection of the curves in $\mathbb{P}^{2}$ given in $\$ 2.2 .1$ reveals that none of them is Galois invariant, hence none are defined over $k$.

Lemma 3.12. Let $P, Q$ be closed points of $\mathbb{P}^{2}$ both of degree 4 , such that $P \sqcup Q$ lies in general position. Assume that the splitting field $K$ of $Q$ is a $V_{4}$ - or $A_{4}$ extension of $k$ which satisfies the conditions of Lemma 2.7. Then $\mathrm{Bl}_{P \sqcup Q} \mathbb{P}^{2}$ is a del Pezzo surface of degree 1 which is a counter-example to the Hasse principle for lines.

Proof. As in the proof of Lemma 3.9, by Lemma 3.11 we know that $S$ contains no line, yet is a blow-up of a del Pezzo surface of degree 5 which is a counter-example to the Hasse principle for lines. The result follows. 
Remark 3.13. The construction given in Lemma 3.12 yields 26 out of the 8742 subgroups of $W\left(\mathbf{E}_{8}\right)$ which satisfy Proposition 3.1. Generically this yields surfaces with orbit type

$$
[4,4,4,4,4,4,4,4,6,6,6,6,12,12,16,16,16,16,24,24,24,24],
$$

and all other surfaces thus obtained have refinements of this orbit type.

3.10.3. A minimal counter-example. We now construct some minimal counterexamples using conic bundles. We consider conic bundles of the shape

$$
z^{2}=f(t) x^{2}+g(t) y^{2} \quad \subset \mathbb{A}^{1} \times \mathbb{P}^{2},
$$

where $f, g \in k[t]$ and $\operatorname{deg} f=\operatorname{deg} g=4$. This is a variant of the family considered in [8, §5]. Given $e_{1}, e_{2}, e_{3}, e_{4}, \lambda, a, b \in k^{*}$, we take $f$ to be

$$
f(t)=\left(t-e_{1}\right)\left(t-e_{2}\right)\left(t-e_{3}\right)\left(t-e_{4}\right),
$$

and also take

$$
g(t)=\lambda f(t)+\prod_{i \neq 1} \frac{t-e_{i}}{e_{1}-e_{i}}+a \prod_{i \neq 2} \frac{t-e_{i}}{e_{2}-e_{i}}+b \prod_{i \neq 3} \frac{t-e_{i}}{e_{3}-e_{i}}+a b \prod_{i \neq 4} \frac{t-e_{i}}{e_{4}-e_{i}} .
$$

This has been defined so that it satisfies

$$
g\left(e_{1}\right)=1, \quad g\left(e_{2}\right)=a, \quad g\left(e_{3}\right)=b, \quad g\left(e_{4}\right)=a b .
$$

With these choices, the fibre over $t=e_{1}$ is split. Contracting one of the lines in this fibre, a similar method to the proof of [8, Prop. 5.3] shows that we obtain a surface with a natural compactification $S$ which is a del Pezzo surface of degree 1 with a conic bundle structure, provided that the $e_{i}$ and $\lambda$ are chosen sufficiently generally. This conic bundle has 7 singular fibres over $\bar{k}$. By construction, three of these fibres occur over rational points, with splitting fields

$$
k(\sqrt{a}), k(\sqrt{b}), k(\sqrt{a b}) .
$$

Moreover if the $e_{i}$ and $\lambda$ are chosen sufficiently generally, then the conic bundle has a non-split fibre over a closed point of degree 4 . Thus the surface is relatively minimal, hence minimal and non-rational by results of Iskovskih ([22, Thm. 4] and [21, Cor. 1.7]). If $a, b$ satisfy the conditions of Lemma 2.8, we see that for each place $v$ of $k$, at least one of the singular fibres splits into a union of lines. Thus we obtain the required counter-example to the Hasse principle for lines.

Remark 3.14. Generically, this construction leads to surfaces with orbit type

$$
[2,2,2,2,2,2,4,4,4,8,8,16,16,16,24,32,32,32,32]
$$

whose Galois group is a solvable group of order 768, and all other surfaces obtained in this way have refinements of this orbit type.

This completes the proof of Theorem 1.4.

\section{Distribution of COUnTER-EXAMPles}

The aim of this section is to prove Theorem 1.3, together with an analogous result for del Pezzo surfaces. We begin by giving the set-up for our results. 
4.1. The set-up. We work for now over $\mathbb{Z}$, and construct a Hilbert scheme which parametrises del Pezzo surfaces of degree $d$. Define

$$
X_{d}= \begin{cases}\mathbb{P}^{d}, & \text { if } d \geq 3, \\ \mathbb{P}(1,1,1,2), & \text { if } d=2, \\ \mathbb{P}(1,1,2,3), & \text { if } d=1\end{cases}
$$

So that any del Pezzo surface of degree $d$ may be embedded anticanonically into $X_{d}$. We define the scheme $\mathcal{H}_{d}$ to be the Hilbert scheme over $\mathbb{Z}$ which parametrises those subschemes of $X_{d}$ which are anticanonically embedded del Pezzo surfaces of degree $d$. This exists by a general result of Grothendieck [18, Exposé 221, Thm. 3.1], and commutes with arbitrary base extensions. Given a $\mathbb{Z}$-scheme $A$, we denote by

$$
\mathcal{H}_{d, A}=\mathcal{H}_{d} \times_{\text {Spec } \mathbb{Z}} A
$$

the Hilbert scheme of anticanonically embedded del Pezzo surfaces of degree $d$ over $A$. We study the basic properties of these schemes in the following lemma.

Lemma 4.1. Let $1 \leq d \leq 9$ and let $k$ be a field. Then the scheme $\mathcal{H}_{d, k}$ is smooth. When $d \neq 8$, it is geometrically connected. When $d=8$, it consists of two connected components, each of which is geometrically connected.

Proof. Both claims are clear when $d \leq 3$, as $\mathcal{H}_{d, k}$ is an open subset of some projective space (it parametrises the smooth members of a linear system of divisors).

Next consider the case $d=4$. Here any such surface is a complete intersection of two quadrics, which is uniquely determined by the pencil of quadrics in $\mathbb{P}^{4}$ which contains it (namely it is the base-locus of such a pencil). Hence $\mathcal{H}_{4, k}$ may be identified with an open subset of the Grassmanian of lines in the projective space $\mathbb{P}\left(H^{0}\left(\mathbb{P}^{4}, \mathcal{O}(2)\right)\right)$, hence is clearly smooth and geometrically connected.

Assume now that $d \geq 5$. In order to prove that $\mathcal{H}_{d, k}$ is smooth, by [18, Exposé 221, Cor. 5.4] it suffices to show that $H^{1}\left(S, N_{S / \mathbb{P}^{d}}\right)=0$ for any smooth del Pezzo surface $S \subset \mathbb{P}^{d}$ of degree $d$, where $N_{S / \mathbb{P}^{d}}$ denotes the normal bundle of $S$ with respect to $\mathbb{P}^{d}$. This vanishing is a standard cohomological calculation, and is shown, for example, in [11, Lem. 5.7].

We now consider the connected components when $d \geq 5$. First suppose that $d \neq 8$. Here there is a unique del Pezzo surface of degree $d$ over $\bar{k}$ up to isomorphism. Thus any two del Pezzo surfaces of degree $d$ in $\mathbb{P}^{d}$ are projectively equivalent over $\bar{k}$, hence $\mathcal{H}_{d, k}$ is a homogeneous space for $\mathrm{PGL}_{d+1}$, and the result follows. When $d=8$, there are exactly two $\bar{k}$-isomorphism classes of del Pezzo surfaces of degree 8 , and each contains a surface defined over $k$. The result then follows from a similar argument to the above.

We denote the two connected components of $\mathcal{H}_{8, k}$ by $\mathcal{H}_{8, k}^{1}$ and $\mathcal{H}_{8, k}^{2}$. We choose these such that $\mathcal{H}_{8, k}^{1}$ parametrises twists of $\mathbb{P}^{1} \times \mathbb{P}^{1}$.

Next, recall (see [38, §9] or [41, §3]) that given a variety $X$ over $k$, a subset $\Omega \subset X(k)$ is said to be thin if it is a finite union of subsets which are either contained in a proper closed subvariety of $X$, or in some $\pi(Y(k))$ where $\pi$ : $Y \rightarrow X$ is a generically finite dominant morphism of degree exceeding 1, with $Y$ irreducible. Given a disjoint union of varieties $X_{1} \sqcup X_{2}$, we shall say that a subset $\Omega \subset\left(X_{1} \sqcup X_{2}\right)(k)$ is thin, if $\Omega \cap X_{i}(k)$ is thin for each $i=1,2$. 
Theorem 4.2. Let $k$ be a number field and let $1 \leq d \leq 9$.

(a) There exists a thin subset $\Omega_{d} \subset \mathcal{H}_{d, k}(k)$ such that the Hasse principle for lines holds for those del Pezzo surfaces corresponding to the points of $\mathcal{H}_{d, k}(k) \backslash \Omega_{d}$.

(b) The collection of del Pezzo surfaces of degree $d$ in $\mathcal{H}_{8, k}^{1}(k)$ which fail the Hasse principle for lines is Zariski dense in $\mathcal{H}_{8, k}^{1}$.

(c) If $d=5,3,2$ or 1 , then the collection of del Pezzo surfaces of degree $d$ in $\mathcal{H}_{d, k}(k)$ which fail the Hasse principle for lines is Zariski dense inside $\mathcal{H}_{d, k}$.

This result says that, whilst there are many counter-examples, they are still in some sense quite rare.

4.2. The universal family of lines. Before we begin the proof of Theorem 4.2 , we study the universal family of lines over the Hilbert schemes $\mathcal{H}_{d}$.

The scheme $\mathcal{H}_{d}$ comes equipped with a universal family of del Pezzo surfaces $\mathcal{S}_{d} \rightarrow \mathcal{H}_{d}$, which naturally lives inside $\mathcal{H}_{d} \times X_{d}$. By a standard argument (see [42, §3.4]), there exists a universal family of lines $\ell_{d}: \mathcal{L}_{d} \rightarrow \mathcal{H}_{d}$, parametrising the lines in the family $\mathcal{S}_{d} \rightarrow \mathcal{H}_{d}$. When $d=8$ and $k$ is a field, we have $\mathcal{H}_{8, k}=$ $\mathcal{H}_{8, k}^{1} \sqcup \mathcal{H}_{8, k}^{2}$ by Lemma 4.1. In which case we denote by $\ell_{8, k}^{1}: \mathcal{L}_{8, k}^{1} \rightarrow \mathcal{H}_{8, k}^{1}$ and $\ell_{8, k}^{2}: \mathcal{L}_{8, k}^{2} \rightarrow \mathcal{H}_{8, k}^{2}$ the corresponding universal families of lines.

Lemma 4.3. Let $1 \leq d \leq 9$ and let $k$ be a field. Then the map

$$
\ell_{d, k}: \mathcal{L}_{d, k} \rightarrow \mathcal{H}_{d, k}
$$

is smooth and projective. If $d \leq 7$, then it is finite étale.

Proof. By construction the morphism $\ell_{d, k}$ is projective. Since $\mathcal{H}_{d, k}$ is reduced by Lemma 4.1, we may apply [20, Thm. III.9.9] to each irreducible component of $\mathcal{H}_{d, k}$ to deduce that $\ell_{d, k}$ is flat. Proposition 2.11 implies that $\ell_{d, k}$ is smooth and quasi-finite when $d \leq 7$. The result then follows since a quasi-finite smooth proper morphism is finite étale (see also [42, Prop. 3.6]).

Proposition 4.4. Let $1 \leq d \leq 9$ and let $k$ be field, with char $k=0$ or char $k \gg 1$. If $d \neq 7,8$, then $\mathcal{L}_{d, k}$ is irreducible. When $d=8$, each of the schemes $\mathcal{L}_{8, k}^{1}$ and $\mathcal{L}_{8, k}^{2}$ is irreducible.

Proof. By a spreading out argument, it suffices to prove the result when $k$ has characteristic 0 . For $d=9,6$ or 5 , we may assume that $k$ is algebraically closed, and use a similar method to Lemma 4.1. Namely, not only is there a unique del Pezzo surface of degree $d$ over $k$, but moreover the automorphism group of the surface acts transitively on the lines (for $d=9$ this is trivial, and for $d=6,5$ this follows from [14, Prop. 8.2.3], [14, Thm. 8.4.2] and [14, Thm. 8.5.6]). As each automorphism of the surface is induced by an automorphism of the ambient projective space, it follows that $\mathcal{L}_{d, k}$ is a homogeneous space for $\mathrm{PGL}_{d+1}$, hence is irreducible. A similar argument applies to give the result when $d=8$. For $d=4$ or 3 , the result is a special case of general results on Hilbert schemes of linear subspaces on complete intersections; see for instance [15, p. 3].

We now consider the case where $d=2$ or 1 . Here, it suffices to prove the result when $k=\overline{\mathbb{Q}}$. However to do this, we may reduce to the case where $k$ is 
an arbitrary number field. As $\ell_{d, k}$ is proper, it suffices to show that there exists a single fibre which is irreducible, i.e. we need a del Pezzo surface $S$ of degree $d$ over $k$ whose Hilbert scheme of lines $\mathcal{L}(S)$ is irreducible. This problem has been considered by numerous authors, and the relevant results already exist in the literature. When $d=1$, the required result over $\mathbb{Q}$ is [50, Thm. 1.3], and moreover [50, Rem. 1.4] gives the result over any number field. When $d=2$, the result over $\mathbb{Q}$ is [17]. The method of [50] is based on the approach used in [17] however, and a minor modification of the argument given at the end of [50, §6], which we do not reproduce here, yields the result over any number field. Both of these results can also be deduced from work of Shioda [43]. This completes the proof.

Remark 4.5. Note that $\mathcal{L}_{7}$ is not irreducible. Indeed, every del Pezzo surface of degree 7 has a distinguished line, thus the morphism $\mathcal{L}_{7} \rightarrow \mathcal{H}_{7}$ admits a section.

4.3. Proof of Theorem 4.2(a). We now assume that $k$ is a number field. Here we begin with the following lemma.

Lemma 4.6. Let $1 \leq d \leq 6$ or $d=8,9$. There exists a thin subset $\Omega_{d} \subset \mathcal{H}_{d, k}(k)$ such that the fibre of $\ell_{d, k}$ over every point of $\mathcal{H}_{d, k}(k) \backslash \Omega_{d}$ is irreducible.

Proof. When $d=9$, the result is trivial (one may take $\Omega_{d}=\emptyset$ by Proposition 2.11). When $d \leq 6$, this follows immediately from Lemma 4.3, Proposition 4.4 and Hilbert's irreducibility theorem [41, Prop. 3.3.1]. When $d=8$, we note that the corresponding result is trivial for $\ell_{8, k}^{2}$. As $\ell_{8, k}^{1}$ is proper by Lemma 4.3, we may consider the Stein factorisation

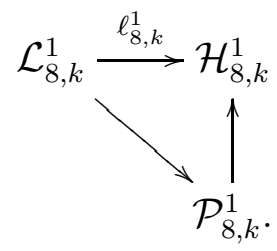

As $\mathcal{L}_{8, k}^{1}$ is irreducible by Proposition 4.4, we see that the scheme $\mathcal{P}_{8, k}^{1}$ is also irreducible. Moreover, by Proposition 2.11, we know that the map $\mathcal{P}_{8, k}^{1} \rightarrow \mathcal{H}_{8, k}^{1}$ is finite étale of degree 2. Therefore the result follows on applying Hilbert's irreducibility theorem to the morphism $\mathcal{P}_{8, k}^{1} \rightarrow \mathcal{H}_{8, k}^{1}$.

Let $S$ be a del Pezzo surface of degree $d$ over $k$ which fails the Hasse principle for lines. By Theorem 1.4 we know that $d=8,5,3,2$ or 1 , and moreover $S$ must be a twist of $\mathbb{P}^{1} \times \mathbb{P}^{1}$ when $d=8$ (see $\$ 3.3$ ). Lemma 2.3 and Lemma 3.3 therefore imply that $\mathcal{L}(S)$ is reducible. Hence, the result follows from Lemma 4.6.

4.4. Proof of Theorem 4.2(b). As explained in 3.3, there exists a del Pezzo surface $S$ of degree 8 which is a twist of $\mathbb{P}^{1} \times \mathbb{P}^{1}$ and which is a counter-example to the Hasse principle for lines. Choose an anticanonical embedding $S \subset \mathbb{P}^{8}$ and identify $S$ with an element of $\mathcal{H}_{8, k}^{1}(k)$. As $\mathcal{H}_{8, k}^{1}$ is a homogeneous space for PGL (see the proof of Lemma 4.1), we see that the orbit of $S$ under the action of $\mathrm{PGL}_{9}(k)$ is Zariski dense in $\mathcal{H}_{8, k}^{1}$, and the result follows. 
4.5. Proof of Theorem 4.2(c). We begin the proof with a lemma which says that, under suitable conditions, one may "approximate" a collection of points in $\mathbb{P}^{2}$ over a finite field by a closed point whose residue field is a pre-specified number field.

Lemma 4.7. Let $L / k$ be a finite field extension of degree $r$, with Galois closure $K / k$. Let $\mathfrak{P}$ be a prime ideal of $K$ which is completely split over $k$ and let $P_{1}, \ldots, P_{r} \in \mathbb{P}^{2}\left(\mathbb{F}_{\mathfrak{P}}\right)$. Then there exists a closed point $P$ of $\mathbb{P}_{k}^{2}$ with residue field $L$ such that

$$
P \equiv P_{1} \sqcup \cdots \sqcup P_{r} \quad \bmod \mathfrak{P} .
$$

Moreover, let $P^{\prime}$ be a closed point of $\mathbb{P}^{2}$ of degree $r^{\prime}$ in general position and assume that $r+r^{\prime} \leq 8$. Then we may choose $P$ such that $P \sqcup P^{\prime}$ lies in general position.

Note that in the statement of the lemma, we are viewing $P$ as a Galois invariant collection of points of $\mathbb{P}^{2}(K)$. In particular, the reduction of $P$ modulo the prime ideal $\mathfrak{P}$ is a well-defined collection of elements of $\mathbb{P}^{2}\left(\mathbb{F}_{\mathfrak{P}}\right)$.

Proof. For $i=1, \ldots, r$, let $\sigma_{i}: L \rightarrow K$ denote the distinct embeddings of $L$ into the field $K$. We are looking for an element $Q \in \mathbb{P}^{2}(L)$ such that

$$
\sigma_{i}(Q) \equiv P_{i} \quad \bmod \mathfrak{P}, \quad \text { for all } i \in\{1, \ldots, r\} .
$$

For each $i$, choose an element $g_{i} \in \operatorname{Gal}(K / k)$ such that $g_{i} \circ \sigma_{i}=\sigma_{1}$. It follows that (4.1) is equivalent to

$$
\sigma_{1}(Q) \equiv g_{i}\left(P_{i}\right) \quad \bmod g_{i}(\mathfrak{P}), \quad \text { for all } i \in\{1, \ldots, r\} .
$$

Next let $\mathfrak{p}_{i}$ denote the distinct prime ideals of $L$ which lie below the $g_{i}(\mathfrak{P})$ and let $Q_{i} \in \mathbb{P}^{2}\left(\mathbb{F}_{\mathfrak{p}_{i}}\right)$ lie below the $g_{i}\left(P_{i}\right)$. We have reduced to finding a $Q$ such that

$$
Q \equiv Q_{i} \quad \bmod \mathfrak{p}_{i}, \quad \text { for all } i \in\{1, \ldots, r\} .
$$

However, the existence of such a $Q$ is now implied by weak approximation for $\mathbb{P}^{2}$ (see for example [44, Thm. 5.1.2]), which implies that the map

$$
\mathbb{P}^{2}(L) \rightarrow \prod_{i=1}^{r} \mathbb{P}^{2}\left(\mathbb{F}_{\mathfrak{p}_{i}}\right),
$$

is surjective. Moreover, simple topological considerations show that we may choose $Q$ such that the general position criterion in the statement of the lemma is satisfied when $r+r^{\prime} \leq 8$. This completes the proof.

We now use this to obtain the following.

Lemma 4.8. Let $d=5,3,2$ or 1 . Then there exist infinitely many prime ideals $\mathfrak{p}$ of $k$ with the following property:

Let $S \subset X_{d}$ be a split anticanonically embedded del Pezzo surface of degree $d$ over $\mathbb{F}_{\mathfrak{p}}$. Then there exists a smooth proper surface $\mathcal{S} \subset X_{d}$, defined over the localisation $\mathcal{O}_{k, \mathfrak{p}}$ of $\mathcal{O}_{k}$ at $\mathfrak{p}$, whose special fibre is equal to $S$, and whose generic fibre is an anticanonically embedded del Pezzo surface of degree $d$ which is a counter-example to the Hasse principle for lines. 
Proof. We first construct $\mathcal{S}$ as an abstract surface, then show how to embed it into $X_{d}$. We do this in detail only in the case where $d=3$; the other cases being handled in a similar manner. We work with the case of $D_{5}$ given in $\$ 3.8 .3$, using the construction of 3.8 .2 .

Choose a quintic extension $K_{2}$ of $k$, whose Galois closure $K$ is a $D_{5}$-extension of $k$ which satisfies the conditions of Lemma 2.7. Let $K_{1}$ denote the unique quadratic subfield of $K$ and let $\mathfrak{p}$ be a prime ideal of $k$ which is completely split in $K$ and whose norm $N(\mathfrak{p})$ is sufficiently large. Finally, let $S \subset X_{3}$ be a split anticanonically embedded del Pezzo surface of degree 3 over $\mathbb{F}_{\mathfrak{p}}$. Blow-up an $\mathbb{F}_{\mathfrak{p}}$-point of $S$ which does not lie on a line, to obtain a del Pezzo surface $S^{\prime}$ of degree 2 (such a point exists as $N(\mathfrak{p})$ is sufficiently large). Denote by $L$ the exceptional curve of the blow-up. Choose pairwise skew lines $L_{1}, \ldots, L_{7}$ on $S^{\prime}$ such that $L_{1}$ and $L_{2}$ each intersect $L$ with multiplicity 1 , but $L_{3}, \ldots, L_{7}$ do not meet $L$. Contracting $L_{1}, \ldots, L_{7}$, we obtain a morphism $S^{\prime} \rightarrow \mathbb{P}^{2}$ together with $\mathbb{F}_{\mathfrak{p}}$-points $P_{1}, \ldots, P_{7}$ given by the images of $L_{1}, \ldots, L_{7}$.

By Lemma 4.7, we may choose closed points $P$ and $Q$ of $\mathbb{P}^{2}$ with residue fields $K_{1}$ and $K_{2}$, respectively, such that $P \sqcup Q$ lies in general position and such that $P$ and $Q$ reduce to $P_{1} \sqcup P_{2}$ and $P_{3} \sqcup \cdots \sqcup P_{7}$ modulo $\mathfrak{p}$, respectively. Let $\mathcal{S}^{\prime}$ denote the surface over $\mathcal{O}_{k, \mathfrak{p}}$ given by the blow-up of $\mathbb{P}^{2}$ in $P$ and $Q$. Contracting the line of $\mathcal{S}^{\prime}$ which reduces to $L$ modulo $\mathfrak{p}$, we obtain a surface $\mathcal{S}$ whose special fibre is isomorphic to $S$. As explained in $\$ 3.8 .3$, the generic fibre of $\mathcal{S}$ is a counterexample to the Hasse principle for lines, as required. This works for any prime ideal $\mathfrak{p}$ which is completely split in $K$ and of sufficiently large norm, in particular, there are infinitely many such prime ideals by Chebotarev's density theorem.

When $d=5,2$ or 1 , the results obtained in $\$ 3.6$, 3.9 and 33.10 imply that we may obtain counter-examples to the Hasse principle for lines by blowing-up certain collections of closed points in $\mathbb{P}^{2}$ with special residue fields. Applying a similar method to the above, we obtain the required surface $\mathcal{S}$ in these cases (in fact here it is even easier, since no contraction is required).

We now show that the embedding of $S$ can be "lifted" to an embedding of $\mathcal{S}$. To prove this, let $\pi: \mathcal{S} \rightarrow$ Spec $R$ be as constructed above, where $R=\mathcal{O}_{k, \mathfrak{p}}$. As $R$ is affine, there is a natural isomorphism

$$
H^{0}\left(R, \pi_{*} \omega_{\mathcal{S} / R}^{-n}\right) \cong H^{0}\left(\mathcal{S}, \omega_{\mathcal{S} / R}^{-n}\right)
$$

of $R$-modules, for each $n \geq 0$. Hence by Grauert's theorem [20, Cor. III.12.9], the reduction modulo $\mathfrak{p}$ map

$$
H^{0}\left(\mathcal{S}, \omega_{\mathcal{S} / R}^{-n}\right) \rightarrow H^{0}\left(S, \omega_{S / \mathbb{F}_{\mathfrak{p}}}^{-n}\right)
$$

is surjective. As the anticanonical embedding of $S$ is determined by a collection of global sections of $H^{0}\left(S, \omega_{S / \mathbb{F}_{\mathfrak{p}}}^{-n}\right)$ for suitable $n \geq 0$, it follows that any anticanonical embedding $S \subset X_{d}$ lifts to an anticanonical embedding $\mathcal{S} \subset X_{d}$, as required.

Let $\mathfrak{p}$ be a prime ideal of $k$. Denote by $C\left(\mathcal{O}_{k, \mathfrak{p}}\right)$ the set of elements of $\mathcal{H}_{d}\left(\mathcal{O}_{k, \mathfrak{p}}\right)$ whose generic fibres are counter-examples to the Hasse principle for lines. Let $C(k) \subset \mathcal{H}_{d}(k)$ and $C\left(\mathbb{F}_{\mathfrak{p}}\right) \subset \mathcal{H}_{d}\left(\mathbb{F}_{\mathfrak{p}}\right)$ denote the generic fibres and special fibres of these elements, respectively. Assume that $C(k)$ is not Zariski dense in $\mathcal{H}_{d, k}(k)$. 
Applying the Lang-Weil estimates [28], we see that

$$
\# C\left(\mathbb{F}_{\mathfrak{p}}\right) \ll N(\mathfrak{p})^{\delta(d)-1}, \quad \text { as } N(\mathfrak{p}) \rightarrow \infty,
$$

where

$$
\delta(d)=\operatorname{dim} \mathcal{H}_{d, k}
$$

However by Lemma 4.8, we know that there are infinitely many prime ideals $\mathfrak{p}$ of $k$ such that

$$
\mathcal{H}_{d}\left(\mathbb{F}_{\mathfrak{p}}\right)_{\text {split }} \subset C\left(\mathbb{F}_{\mathfrak{p}}\right)
$$

where $\mathcal{H}_{d}\left(\mathbb{F}_{\mathfrak{p}}\right)_{\text {split }}$ denotes the collection of elements of $\mathcal{H}_{d}\left(\mathbb{F}_{\mathfrak{p}}\right)$ which correspond to split surfaces. Therefore to complete the proof of Theorem 4.2 (c), it suffices to show the following.

Lemma 4.9. Let $1 \leq d \leq 7$ and let $q$ be a power of a sufficiently large prime. Then

$$
\# \mathcal{H}_{d}\left(\mathbb{F}_{q}\right)_{\text {split }} \gg q^{\delta(d)}, \quad \text { as } q \rightarrow \infty .
$$

Proof. By the definition of $\mathcal{L}_{d}$, we have

$$
\mathcal{H}_{d}\left(\mathbb{F}_{q}\right)_{\text {split }}=\ell_{d}\left(\mathcal{L}_{d}\left(\mathbb{F}_{q}\right)\right) .
$$

As $\ell_{d, \mathbb{F}_{q}}$ is finite étale by Lemma 4.3, it therefore suffices to show that

$$
\# \mathcal{L}_{d}\left(\mathbb{F}_{q}\right) \gg q^{\delta(d)} \text {, as } q \rightarrow \infty \text {. }
$$

However again since $\ell_{d, \mathbb{F}_{q}}$ is finite étale, we have $\operatorname{dim} \mathcal{L}_{d, \mathbb{F}_{q}}=\delta(d)$ for $q \gg 1$. As $\mathcal{L}_{d, \mathbb{F}_{q}}$ is geometrically integral for $q$ sufficiently large by Proposition 4.4, the result follows from applying the Lang-Weil estimates [28] to $\mathcal{L}_{d, \mathbb{F}_{q}}$.

This completes the proof of Theorem 4.2(c), hence the proof of Theorem 4.2.

4.6. Proof of Theorem 1.3. Here $\mathcal{H}_{3} \subset \mathbb{P}^{19}$ is the open subset given by the complement of the discriminant locus. Theorem 1.3(a) follows from Theorem 4.2(c). Next by Theorem 4.2(a), we know that the counter-examples to the Hasse principle for lines lie on a thin subset. Theorem 1.3(b) therefore follows from the fact that $0 \%$ of rational points of bounded height lie inside any given thin subset of projective space (see [38, Thm. 13.3]). The result is proved.

\section{INTERSECTIONS OF TWO QUADRICS}

The aim of this section is to prove Theorem 1.5. We begin with some results on the geometry of intersections of two quadrics and pencils of quadrics. For background see [36] or [51, §3.3].

5.1. Geometry of intersections of two quadrics. Let $n \geq 0$ be an integer and let $k$ be a perfect field of characteristic not equal to 2 . Let $X$ be a smooth complete intersection of two quadrics over $k$ in $\mathbb{P}^{2 n+2}$. Denote by $\mathcal{L}(X)$ the Hilbert scheme of $n$-planes inside $X$, which is a finite scheme of degree $2^{2 n+2}$. Note that when $X$ is a del Pezzo surface, this agrees with the definition given in $\$ 2.2 .2$. For these facts, and more, see [36].

The aim of this section is to construct the action of a certain group scheme $\mathrm{R}_{K / k}\left(\mu_{2}\right) / \mu_{2}$ on $X$, where $\mathrm{R}_{K / k}$ denotes the Weil restriction, whose induced action on $\mathcal{L}(X)$ is simply transitive (i.e. such that $\mathcal{L}(X)$ is a torsor under 
$\left.\mathrm{R}_{K / k}\left(\mu_{2}\right) / \mu_{2}\right)$. We shall do this by using pencils of quadrics and descent. The constructions we shall use are generalisations of some of the constructions used in [45], where the author only considers the case of del Pezzo surfaces of degree 4. Suppose that $X$ is defined by the equations

$$
Q_{1}(x)=Q_{2}(x)=0 \quad \subset \mathbb{P}^{2 n+2} .
$$

Consider the pencil of quadrics containing $X$, given as the subvariety

$$
\lambda Q_{1}(x)+\mu Q_{2}(x)=0 \quad \subset \mathbb{P}^{1} \times \mathbb{P}^{2 n+2} .
$$

To this we associate the discriminant

$$
\operatorname{det}\left(\lambda Q_{1}+\mu Q_{2}\right)=0 \quad \subset \mathbb{P}^{1},
$$

which parametrises the singular quadrics in the pencil. Note that here we abuse notation, and identify each $Q_{i}$ with the corresponding symmetric matrix. As $X$ is smooth, the discriminant is a finite reduced closed subscheme of $\mathbb{P}^{1}$ of degree $2 n+3$ [51, Prop. 3.26]. Let $K$ denote the associated finite étale $k$-algebra.

In order to construct the associated group scheme, we first work over $\bar{k}$. Each singular quadric in the pencil over $\bar{k}$ is a cone over a smooth quadric of dimension $2 n$, and moreover the singular point of the quadric does not lie on $X_{\bar{k}}[51$, Lem. 3.7]. Projecting away from the singular point, one realises $X_{\bar{k}}$ as a double cover of the associated smooth quadric. The deck transformation of this cover is then an involution defined on $X_{\bar{k}}$. Considering the group generated by these involutions, we obtain an action of the group $(\mathbb{Z} / 2 \mathbb{Z})^{2 n+3}$ on $X_{\bar{k}}$. All this data is Galois invariant, hence descends to $k$. We therefore obtain the action of a group scheme on $X$ over $k$, which is easily seen to be isomorphic to $\mathrm{R}_{K / k}\left(\mu_{2}\right)$. This does not act faithfully on $X$, however the quotient $\mathrm{R}_{K / k}\left(\mu_{2}\right) / \mu_{2}$ does act faithfully.

Proposition 5.1. Let $X$ be a smooth $2 n$-dimensional complete intersection of two quadrics over $k$ and let $\mathrm{R}_{K / k}\left(\mu_{2}\right) / \mu_{2}$ be the associated group scheme, as constructed above. Then $\mathcal{L}(X)$ is a torsor under $\mathrm{R}_{K / k}\left(\mu_{2}\right) / \mu_{2}$.

Proof. To prove the result we may assume that $k$ is algebraically closed. In which case, this is [36, Thm. 3.8].

\subsection{Proof of Theorem 1.5.}

5.2.1. Tate-Shafarevich groups. We begin the proof by recalling some facts about Tate-Shafarevich groups of commutative group schemes. Let $k$ be a number field and $S$ be a finite set of places of $k$. For a commutative group scheme $G$ over $k$, denote by

$$
\amalg_{S}(k, G)=\operatorname{ker}\left(H^{1}(k, G) \rightarrow \prod_{\substack{v \in \operatorname{Val}(k) \\ v \notin S}} H^{1}\left(k_{v}, G\right)\right),
$$

the $S$-Tate-Shafarevich group of $G$. Here $H^{1}(k, G)$ classifies torsors of $G$ up to isomorphism, with a torsor having the trivial class if and only if it has a rational point (see for example [44, §2.1]). In particular, the non-trivial elements of $\amalg_{S}(k, G)$ classify those torsors of $G$ which are locally soluble outside of $S$. When $S=\emptyset$, such torsors are exactly those which fail the Hasse principle. 
5.2.2. Proof of Theorem 1.5. Given Proposition [5.1, in order to prove Theorem 1.5 it suffices to show the following.

Proposition 5.2. Let $k$ be a number field and let $S$ be a finite set of places of $k$. Let $K$ be an étale $k$-algebra of odd degree. Then

$$
\amalg_{S}\left(k, \mathrm{R}_{K / k}\left(\mu_{2}\right) / \mu_{2}\right)=0 .
$$

Proof. First note that since $K / k$ has odd degree, the composed morphism

$$
\mathrm{R}_{K / k}^{1}\left(\mu_{2}\right) \rightarrow \mathrm{R}_{K / k}\left(\mu_{2}\right) \rightarrow \mathrm{R}_{K / k}\left(\mu_{2}\right) / \mu_{2},
$$

is an isomorphism, where $\mathrm{R}_{K / k}^{1}\left(\mu_{2}\right)$ denotes the norm 1 subgroup scheme of $\mathrm{R}_{K / k}\left(\mu_{2}\right)$. From which it follows that the sequence

$$
1 \rightarrow \mu_{2} \rightarrow \mathrm{R}_{K / k}\left(\mu_{2}\right) \rightarrow \mathrm{R}_{K / k}\left(\mu_{2}\right) / \mu_{2} \rightarrow 1,
$$

is a split short exact sequence of group schemes. Hence we obtain an embedding

$$
\amalg_{S}\left(k, \mathrm{R}_{K / k}\left(\mu_{2}\right) / \mu_{2}\right) \hookrightarrow \amalg_{S}\left(k, \mathrm{R}_{K / k}\left(\mu_{2}\right)\right) .
$$

Write $K=\prod_{i=1}^{r} K_{i}$, where the $K_{i}$ are field extensions of $k$ and let $S_{i}$ denote the set of places of $K_{i}$ which lie above the places of $S$. Then by Shapiro's lemma [39, Prop. 2.5.10] it suffices to show that $\amalg_{S_{i}}\left(K_{i}, \mu_{2}\right)=0$ for each $i \in\{1, \ldots, r\}$. However $H^{1}\left(K_{i}, \mu_{2}\right)$ classifies quadratic extensions of $K_{i}$, hence this vanishing follows from the fact that any quadratic extension is non-split at infinitely many places. This proves the result.

This completes the proof of Theorem 1.5.

\section{REFERENCES}

[1] B. Banwait and J. Cremona, Tetrahedral elliptic curves and the local-to-global principle for isogenies. Algebra and Number Theory 8 (2014), no. 5, 1201-1229.

[2] D. Berend and Y. Bilu, Polynomials with roots modulo every integer. Proc. AMS. 124 (1996), no. 6, 1663-1671.

[3] B. J. Birch, Homogeneous forms of odd degree in a large number of variables. Mathematika 4 (1957), no. 2, 102-105.

[4] W. Bosma, J. Cannon and C. Playoust, The Magma algebra system. I. The user language. J. Symbolic Comput., 24 (1997), no. 3-4, 235-265.

[5] N. Bourbaki, Éléments de mathématique. Algèbre. Chapitres 4 à 7 . Lecture Notes in Mathematics, 864. Masson, Paris, 1981.

[6] J. Bourgain and A. Kontorovich, On the local-global conjecture for integral Apollonian gaskets. Invent. Math. 196 (2014), no. 3, 589-650.

[7] J. Brandes, Forms representing forms and linear spaces on hypersurfaces. Proc. Lond. Math. Soc. 108 (2014), no. 4, 809-835.

[8] T. D. Browning, L. Matthiesen and A. Skorobogatov, Rational points on pencils of conics and quadrics with many degenerate fibres. Annals of Math. 180 (2014), no. 1, 381-402.

[9] J.-L. Colliot-Thélène, J.-J. Sansuc and P. Swinnerton-Dyer, Intersections of two quadrics and Châtelet surfaces. II. J. Reine Angew. Math. 374 (1987), 72-168.

[10] D. Coray and C. Manoil, On large Picard groups and the Hasse principle for curves and K3 surface. Acta Arith. 76 (1996), no. 2, 165-189.

[11] I. Coskun, The enumerative geometry of Del Pezzo surfaces via degenerations. Amer. J. Math. 128 (2006), no. 3, 751-786.

[12] R. Dietmann, Linear spaces on rational hypersurfaces of odd degree. Bull. Lond. Math. Soc. 42 (2010), no. 5, 891-895. 
[13] R. Dietmann and T. Wooley, Pairs of cubic forms in many variables. Acta Arith. 110 (2003), no. 2, 125-140.

[14] I. Dolgachev, Classical algebraic geometry. A modern view. Cambridge University Press, Cambridge, 2012.

[15] O. Debarre and L. Manivel, Sur la variété des espaces linéaires contenus dans une intersection complète. Math. Ann. 312 (1998), no. 3, 549-574.

[16] A.-S. Elsenhans and J. Jahnel, Cubic surfaces with a Galois invariant double-six. Central European Journal of Mathematics 8 (2010), no. 4, 646-661.

[17] R. Erné, Construction of a del Pezzo surface with maximal Galois action on its Picard group. J. Pure Appl. Algebra 97 (1994), no. 1, 15-27.

[18] A. Grothendieck, Fondements de la géométrie algébrique. [Extraits du Séminaire Bourbaki, 1957-1962]. Secrétariat mathématique, Paris 1962.

[19] C. F. Geiser, Ueber die Doppeltangenten einer ebenen Curve vierten Grades. Math. Ann. 1 (1869), no. 1, 129-138.

[20] R. Hartshorne, Algebraic Geometry. Springer-Verlag, 1977.

[21] V. A. Iskovskih, Rational surfaces with a sheaf of rational curves and with a positive square of canonical class. Mat. Sb. (N.S.) 83(125) (1970), 90-119.

[22] V. A. Iskovskih, Minimal models of rational surfaces over arbitrary fields. Izv. Akad. Nauk SSSR Ser. Mat. 43 (1979), no. 1, 19-43, 237.

[23] K. S. Kedlaya, A construction of polynomials with squarefree discriminants. Proc. AMS. 140 (2012), no. 12, 3025-3033.

[24] J. Klüners, G. Malle, A database for field extensions of the rationals. LMS. J. Comput. Math. 4 (2001), 182-196.

[25] J. Kollár, Rational curves on algebraic varieties. A Series of Modern Surveys in Mathematics 32. Springer-Verlag, Berlin, 1996.

[26] J. Kollár, Polynomials with integral coefficients, equivalent to a given polynomial. Electron. Res. Announc. AMS. 3 (1997), 17-27.

[27] S. Lang, Algebra. Revised third edition. Graduate Texts in Mathematics, 211. SpringerVerlag, New York, 2002.

[28] S. Lang and A. Weil, Number of points of varieties in finite fields. Amer. J. Math. 76, no. $4,(1954), 819-827$

[29] D. B. Leep, Systems of quadratic forms. J. Reine Angew. Math. 350 (1987), 109-116.

[30] Q. Liu, Algebraic geometry and arithmetic curves. Oxford Graduate Texts in Mathematics, 6. Oxford Science Publications. Oxford University Press, Oxford, 2002.

[31] Y. I. Manin, Cubic Forms: Algebra, geometry, arithmetic. North-Holland Mathematical Library 4, North-Holland Publishing Co., 2nd ed. 1986.

[32] G. Martin, Solubility of systems of quadratic forms. Bull. Lond. Math. Soc. 29 (1997), no. $4,385-388$.

[33] W. Narkiewicz, Elementary and analytic theory of algebraic numbers. Third edition. Springer Monographs in Mathematics. Springer-Verlag, Berlin, 2004.

[34] J. Neukirch, Algebraic number theory. Springer-Verlag, Berlin, 1999.

[35] O. T. O'Meara, Introduction to Quadratic Forms. Corr. 3rd printing. Springer-Verlag, Berlin, 1973.

[36] M. Reid, The complete intersection of two or more quadrics. Ph.D. thesis.

[37] J.-P. Serre, Local Fields. Springer-Verlag, Berlin, 1980.

[38] J.-P. Serre, Lectures on the Mordell-Weil theorem. 3rd ed., Aspects of Mathematics, Friedr. Vieweg \& Sohn, Braunschweig, 1997.

[39] J.-P. Serre, Galois cohomology, corrected ed. Springer, 1997.

[40] J.-P. Serre, On a theorem of Jordan. Bull. AMS. 40 (2003), no. 4, 429-440.

[41] J.-P. Serre, Topics in Galois theory. Second edition. Research Notes in Mathematics 1. A K Peters, Ltd., Wellesley, MA, 2008.

[42] A.-J. Scholl, A finiteness theorem for del Pezzo surfaces over algebraic number fields. $J$. Lond. Math. Soc. 32 (1985), no. 1, 31-40. 
[43] T. Shioda, Theory of Mordell-Weil lattices. Proceedings of the International Congress of Mathematicians, Vol. I, II (Kyoto, 1990), 473-489, Math. Soc. Japan, Tokyo, 1991.

[44] A. Skorobogatov, Torsors and rational points. Cambridge University press, 2001.

[45] A. Skorobogatov, Del Pezzo surfaces of degree 4 and their relation to Kummer surfaces. Enseign. Math., 56 (2010), no. 1-2, 73-85.

[46] J. Sonn, Polynomials with roots in $\mathbb{Q}_{p}$ for all p. Proc. AMS. 136 (2008), no. 6, 1955-1960.

[47] J. Sonn, Two remarks on the inverse Galois problem for intersective polynomials. J. Th. Nombres de Bordeaux 21 (2009), no. 2, 437-439.

[48] A. Sutherland, A local-global principle for rational isogenies of prime degree. J. Th. Nombres de Bordeaux 24 (2012), no. 2, 475-485.

[49] M. Stoll, Finite descent obstructions and rational points on curves. Algebra and Number Theory 1 (2007), no. 4, 349-391.

[50] A. Várilly-Alvarado and D. Zywina, Arithmetic $E_{8}$ lattices with maximal Galois action. LMS J. Comput. Math. 12 (2009), $144-165$.

[51] O. Wittenberg, Intersections de deux quadriques et pinceaux de courbes de genre 1. Lecture Notes in Mathematics, 1901. Springer, Berlin, 2007.

Jörg Jahnel, Department Mathematik, Walter-Flex-Strasse 3, Universität Siegen, D-57072, Siegen, Germany.

E-mail address: jahnel@mathematik.uni-siegen.de

URL: http://www.uni-math.gwdg.de/jahnel

Daniel Loughran, Leibniz Universität Hannover, Institut für Algebra, ZahLentheorie und Diskrete Mathematik, Welfengarten 1, 30167 Hannover, GerMANY.

E-mail address: loughran@math.uni-hannover.de

$U R L:$ http://www.iazd.uni-hannover.de/ loughran/ 